\title{
Use, adoption, and effectiveness of tippy- tap handwashing station in promoting hand hygiene practices in resource-limited settings: a systematic review
}

Balwani Chingatichifwe Mbakaya ${ }^{1 *}$ (D), Fatch Welcome Kalembo ${ }^{2}$ and Maggie Zgambo ${ }^{1}$

\begin{abstract}
Background: Tippy-taps are locally made devices for washing hands with running water. They are simple and lowcost, enabling technology that provides adequate water sources, handwashing stations and motivation for people to prioritise handwashing. This systematic review aimed to establish the use, benefits, adoption and effectiveness of enabling technology; tippy-tap handwashing station, in resource-limited settings.

Methods: We systematically searched for articles in the PubMed, EMBASE, PsycINFO, AMED, CINAHL, DOAJ and Google Scholar databases guided by the acceptable best practice developed by the PROSPERO and COCHRANE for systematic search and selection of articles. Search terms such as tippy-taps, enabling technology, hand-washing station, hand-washing behaviour, diarrhoea, respiratory infection, increase handwashing behaviour were used. In addition, a PRISMA flow diagram was used to elaborate on the number of articles retrieved, retained, excluded and reasons for every action. Studies that used tippy-tap hand washing station as a handwashing facility regardless of the design were included in this review. A mixed method appraisal tool was used to appraise studies.

Results: Twenty articles met the eligibility criteria. The use of tippy-taps for handwashing by household members or school children was reported by authors of 16 studies, and it ranged from 2.7 to $80 \%$. The availability of tippytaps increased handwashing and use of soap among participants. Furthermore, the majority of people who were oriented to tippy-taps or recruited to tippy-tap studies built their tippy-tap stations even after the promotional activities or programs had ended. In one study, tippy-taps were reported by participant to be effective in preventing episodes of stomach pain among participants.
\end{abstract}

Conclusion: Tippy-tap handwashing station could help in promoting handwashing practice in resource constraint settings. Future studies are needed to evaluate the effectiveness of tippy-tap hand washing station on preventing water and hygiene-related infections.

Keywords: Tippy-tap, Handwashing station, Adoption, Effectiveness, Hand hygiene practice

\footnotetext{
* Correspondence: bcmbakaya@gmail.com

${ }^{1}$ St John's Institute for Health, P.O. Box 18, Mzuzu, Malawi

Full list of author information is available at the end of the article
}

(c) The Author(s). 2020 Open Access This article is licensed under a Creative Commons Attribution 4.0 International License, which permits use, sharing, adaptation, distribution and reproduction in any medium or format, as long as you give appropriate credit to the original author(s) and the source, provide a link to the Creative Commons licence, and indicate if changes were made. The images or other third party material in this article are included in the article's Creative Commons licence, unless indicated otherwise in a credit line to the material. If material is not included in the article's Creative Commons licence and your intended use is not permitted by statutory regulation or exceeds the permitted use, you will need to obtain permission directly from the copyright holder. To view a copy of this licence, visit http://creativecommons.org/licenses/by/4.0/. The Creative Commons Public Domain Dedication waiver (http://creativecommons.org/publicdomain/zero/1.0/) applies to the data made available in this article, unless otherwise stated in a credit line to the data. 


\section{Background}

The United Nations International Children's Emergency Fund (UNICEF) estimate that 884 million people in the world lack access to basic drinking water supply services [1]. The majority of these people live in rural areas of low and middle-income countries [1]. Lack of improved water sources in these areas is problematic not only to the households but also to the public facilities such as hospitals and schools [2]. The World Health Organisation (WHO) states that $38 \%$ of healthcare facilities lack an improved water source, 19\% lack improved sanitation, and $35 \%$ lack water and soap for handwashing in developing countries [2]. In addition, more than half of all primary schools in developing countries do not have adequate water facilities and nearly two-thirds lack adequate sanitation [1]. Where water or water stations are not readily available, neglecting hand washing is not uncommon. Failure to wash hands after visiting the toilet, before eating or feeding a child, before and after preparing food, and after changing and cleaning up a child who has used a toilet, increases the risk of contracting or spreading diarrheal and respiratory-related diseases $[2,3])$. The inadequacy of water supply, sanitation and hygiene cause the death of a child every minute, $80 \%$ of childhood diseases, 272 million days of school absenteeism and other health conditions such as diarrhoea and respiratory disorders in the general population $[4,5]$.

Although lack of resources and modern technology are commonly associated with the inadequate handwashing stations, low cost and simple handwashing and technology such as tippy-taps may provide adequate water sources, stations and motivation for people to prioritise handwashing [6]. Tippy-taps are simple and economic handwashing stations, made with locally available materials including plastic containers, jerry cans or gourds, and do not depend on a piped water supply [6]. Biran [7] describes a tippy-tap as 'a device consisting of a small (three or five-litre) jerry can be filled with water and suspended from a wooden frame. A string is attached to the neck of the jerry can that can be tied to a piece of wood at ground level. Pressing on this piece of wood with the foot, tips the jerry can to release a stream of water through a small hole. Soap is suspended from the frame beside the jerry can' (See Figs. 1 and 2). Furthermore, tippy-taps are easy to construct, use very little water, easier to use and only soap is touched, thereby making handwashing very hygienic because it avoids contamination of the jerry can, unlike the real tap [7]. Tippy-taps could be a technology of choice for reducing diarrheal and respiratory disorders and deaths that are associated with lack of water, inadequate handwashing stations and practices through controlling factors that hinder handwashing practice such as unavailability of handwashing station, water and soap [10]. Following this, it should,

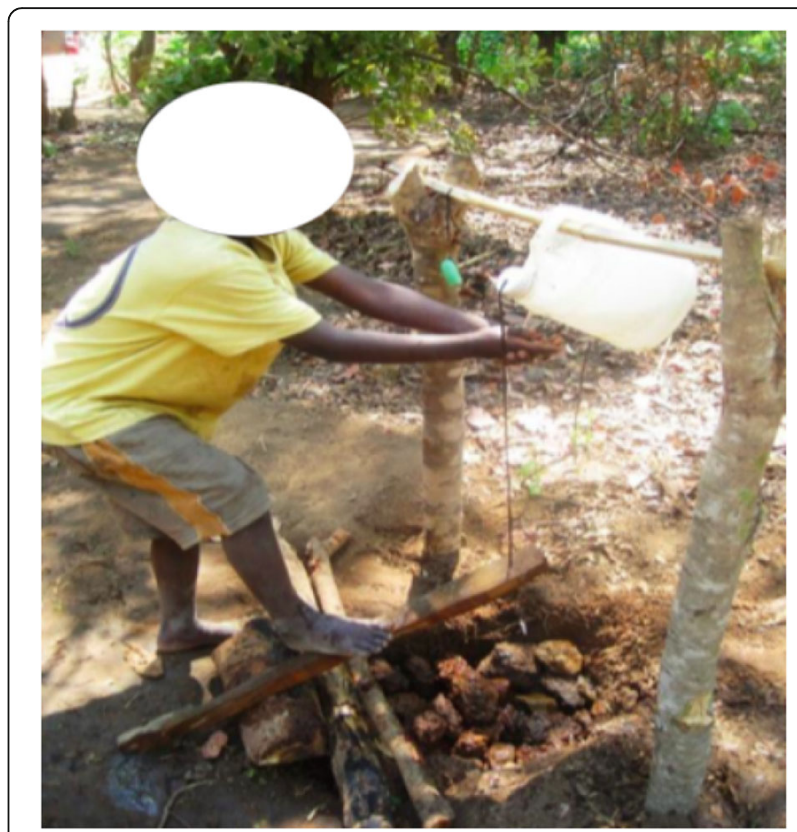

Fig. 1 A boy washing hands using tippy-tap. Source: UNICEF/Zambia/2012/Asindua [8]

therefore, be noted that reducing infectious diseases that occur due to unhygienic hand practices takes more that handwashing education, the handwashing stations, water and soap equally play a major role in reducing.

The first tippy-tap was constructed by Dr. Jim Watt and Jackson Masawi of the Salvation Army in Chiweshe, Zimbabwe, and was called the Mukombe in the 1980s.

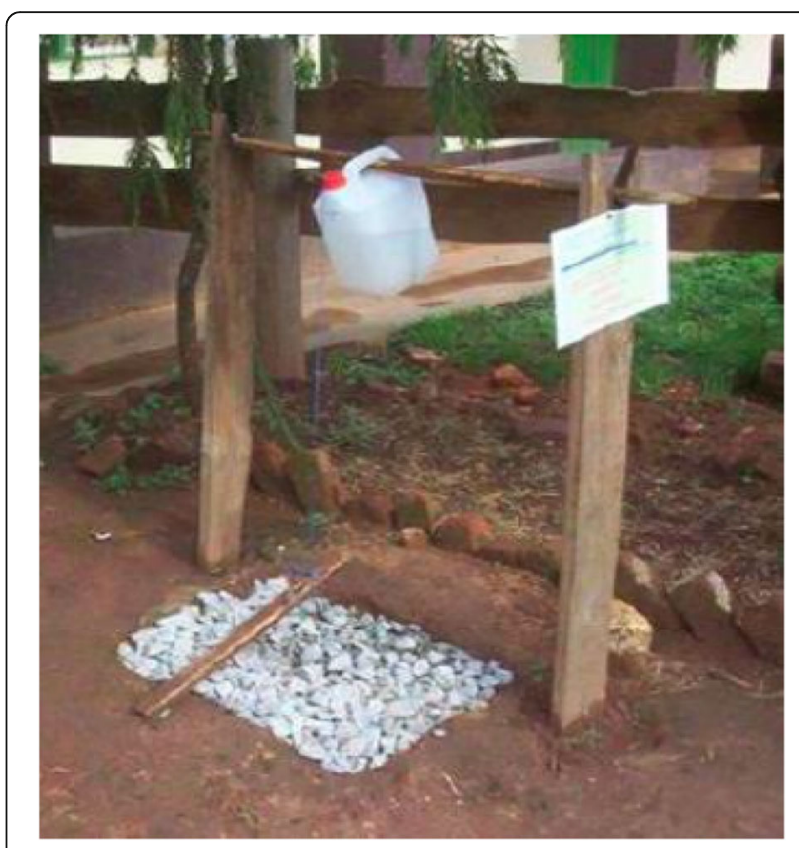

Fig. 2 An examples of a tippy-tap. Source: Mark Tiele Westra [9] 
The Mukombe is a type of gourd or calabash, which is used as the can [11]. Since then, many different versions of tippy-taps have emerged in different parts of the world, depending on the accessibility and types of available local materials. Tippy-taps, although simply constructed from locally affordable and accessible materials, could be the suitable handwashing stations for underdeveloped settings that often lack adequate water for handwashing. The average amount of water used for handwashing using tippy-taps is far much less compared to ordinary handwashing stations such as taps. Comparatively, a good hand wash using tippy-tap could use only $50 \mathrm{mls}$ of water, while washing hands using tap water may utilise up to $500 \mathrm{mls}$ of water [11]. Furthermore, tippy-taps could help to increase handwashing behaviour in schools because it is appealing to children since it is humorous and easy to use, consequently cutting the number of deaths in children that occur due to health conditions associated with hand hygiene practices [7]. Enabling technology is one of the factors that externally influence individual's probability to accomplish a behaviour [7]. The UNICEF and WaterAid recommend the use of tippy-taps in schools and family houses next to the latrines $[12,13]$. Tippy-tap is possibly the best known low cost enabling technology for handwashing [7] and currently, tippy-taps are commonly used in East and Southern Africa in countries like Uganda, Rwanda, and Zambia [13].

The aim of this systematic review, therefore, was to gather, consolidate and quantify the evidence of the use, benefits, adoption and effectiveness of tippy-tap handwashing station in promoting hand hygiene practices in resource-limited settings. Promotion of handwashing behaviour was the main outcome in this systematic review. The secondary outcomes were use, adoption, benefits and effectiveness of tippy-taps. The questions that were addressed by this review are: 1) How does the use of tippy-tap handwashing stations promote hand hygiene practices in a resource-limited setting? 2) How effective are tippy-taps in promoting hand hygiene and reducing water and hygiene-related infections?

\section{Methods}

\section{Protocol}

This review was guided by the acceptable best practice developed by the PROSPERO and COCHRANE for systematic search and selection of articles. The protocol was published in the PROSPERO database with registration number CRD42017074331 [14].

\section{Inclusion criteria}

All studies that used tippy-tap handwashing station as a handwashing facility regardless of the design were included in this systematic review.

\section{Exclusion criteria}

Papers written in languages other than English and articles with studies conducted in developed countries were excluded.

\section{Information source /search strategy}

The following database sources were used to gather the required information; Medline, EMBASE, PsycINFO, AMED, CINAHL, DOAJ and Google Scholar. MeSH terms such as hand hygiene, hand disinfection, hand washing, handwashing, hand washings, washings, hand scrubbing, scrubbing, infection, cross-infection, waterborne, waterborne disease, water related diseases, water diseases and diarrhoea were used during searching for the articles to ensure accuracy. Besides MeSH terms, keywords were also combined using Boolean operators $\mathrm{OR}$ and AND. The following key terms and MeSH terms were used: Tippy-taps, OR Enabling technology OR Hand-washing station OR Hand washing interventions OR Hand washing strategies OR Hand washing programs AND Hand wash OR Hand washing OR Hand washings OR Handwashing OR Hand washing behaviour, OR Hand washing techniques OR Hand hygiene OR Hand disinfection OR Hand or Washings OR Hand scrubbing AND Use OR Usefulness OR Utilisation OR Benefit OR Advantages OR Effectiveness OR evaluation AND Promotion OR Sustainability OR Adoption OR Appropriateness AND Prevention OR Control OR Limit AND diarrhoea, OR dysentery OR waterborne disease OR bloody stool OR Loose stool OR Respiratory Infection OR Infection OR Cross infection (see Table 1). Keywords were also used to search for articles in Google Scholar. Efforts were made to identify both published and unpublished interventional studies by manually checking the reference list of the articles that met the inclusion criteria. Several strategies were used to identify unpublished studies. First, we reviewed the methodology and reference list of the included studies to assess if they identified any unpublished research related to the review question. Second, we manually searched conference proceedings such as Development International Conference, Water Engineering and Development Centre and the University of North Caroline Water and Health Conference for any suitable studies. Further searches were conducted in clinical trial website such as ClinicalTrials.gov website (https://clinicaltrials.gov/). Efforts were also made to contact the authors of the unpublished studies. Reference lists of the included studies were checked and hand searching in the key journals was also done. The search period for the research articles in the mentioned databases was from the inception of the databases to July 2019. The search for the eligible studies in the database was conducted between September 2017 to July 2019. 
Table 1 Search strategy

\begin{tabular}{|c|c|c|c|}
\hline Databases & Search & Search words/terms & Results \\
\hline$\overline{\mathrm{CINAHL}}$ & Title \& abstract & $\begin{array}{l}\text { Tippy-taps, OR Enabling technology OR Hand-washing station OR Hand washing interventions OR } \\
\text { Hand washing strategies OR Hand washing programs AND Hand wash OR Hand washing OR Hand } \\
\text { washings OR Handwashing OR Hand washing behaviour, OR Hand washing techniques OR Hand } \\
\text { hygiene OR Hand disinfection OR Hand or Washings OR Hand scrubbing AND Use OR Usefulness } \\
\text { OR Utilisation OR Benefit OR Advantages OR Effectiveness OR evaluation AND Promotion OR } \\
\text { Sustainability Or Adoption OR Appropriateness AND Prevention OR Control OR Limit AND diarrhoea, } \\
\text { OR dysentery OR waterborne disease OR bloody stool OR Loose stool OR Respiratory Infection OR } \\
\text { Infection OR Cross infection }\end{array}$ & 4 \\
\hline
\end{tabular}

MEDLINE

AMED

PsychINFO

DOA

Google Scholar EMBASE searched

Total articles included
Title \& abstract Tippy-taps, OR Enabling technology OR Hand-washing station OR Hand washing interventions OR Hand washing strategies OR Hand washing programs AND Hand wash OR Hand washing OR Hand washings OR Handwashing OR Hand washing behaviour, OR Hand washing techniques OR Hand hygiene OR Hand disinfection OR Hand or Washings OR Hand scrubbing AND Use OR Usefulness OR Utilisation OR Benefit OR Advantages OR Effectiveness OR evaluation AND Promotion OR Sustainability Or Adoption OR Appropriateness AND Prevention OR Control OR Limit AND diarrhoea, OR dysentery OR waterborne disease OR bloody stool OR Loose stool OR Respiratory Infection OR Infection OR Cross infection

Title \& abstract Tippy-taps, OR Enabling technology OR Hand-washing station OR Hand washing interventions OR Hand washing strategies OR Hand washing programs AND Hand wash OR Hand washing OR Hand washings OR Handwashing OR Hand washing behaviour, OR Hand washing techniques OR Hand hygiene OR Hand disinfection OR Hand or Washings OR Hand scrubbing AND Use OR Usefulness OR Utilisation OR Benefit OR Advantages OR Effectiveness OR evaluation AND Promotion OR Sustainability Or Adoption OR Appropriateness AND Prevention OR Control OR Limit AND diarrhoea, OR dysentery OR waterborne disease OR bloody stool OR Loose stool OR Respiratory Infection OR Infection OR Cross infection

Title, abstract \& Tippy-taps, OR Enabling technology OR Hand-washing station OR Hand washing interventions OR full article Hand washing strategies OR Hand washing programs AND Hand wash OR Hand washing OR Hand washings OR Handwashing OR Hand washing behaviour, OR Hand washing techniques OR Hand hygiene OR Hand disinfection OR Hand or Washings OR Hand scrubbing AND Use OR Usefulness OR Utilisation OR Benefit OR Advantages OR Effectiveness OR evaluation AND Promotion OR Sustainability Or Adoption OR Appropriateness AND Prevention OR Control OR Limit AND diarrhoea, OR dysentery OR waterborne disease OR bloody stool OR Loose stool OR Respiratory Infection OR Infection OR Cross infection

Title, abstract \& Tippy-taps, OR Enabling technology OR Hand-washing station OR Hand washing interventions OR full article Hand washing strategies OR Hand washing programs AND Hand wash OR Hand washing OR Hand washings OR Handwashing OR Hand washing behaviour, OR Hand washing techniques OR Hand hygiene OR Hand disinfection OR Hand or Washings OR Hand scrubbing AND Use OR Usefulness OR Utilisation OR Benefit OR Advantages OR Effectiveness OR evaluation AND Promotion OR Sustainability Or Adoption OR Appropriateness AND Prevention OR Control OR Limit AND diarrhoea, OR dysentery OR waterborne disease OR bloody stool OR Loose stool OR Respiratory Infection OR Infection OR Cross infection

Title \& abstract Tippy-taps and handwashing

Title, abstract \& Tippy-taps, OR Enabling technology OR Hand-washing station OR Hand washing interventions OR full article Hand washing strategies OR Hand washing programs AND Hand wash OR Hand washing OR Hand washings OR Handwashing OR Hand washing behaviour, OR Hand washing techniques OR Hand hygiene OR Hand disinfection OR Hand or Washings OR Hand scrubbing AND Use OR Usefulness OR Utilisation OR Benefit OR Advantages OR Effectiveness OR evaluation AND Promotion OR Sustainability Or Adoption OR Appropriateness AND Prevention OR Control OR Limit AND diarrhoea, OR dysentery OR waterborne disease OR bloody stool OR Loose stool OR Respiratory Infection OR Infection OR Cross infection

Reference search from Title, abstract \& Tippy-taps, OR Enabling technology OR Hand-washing station OR Hand washing interventions OR other sources full article Hand washing strategies OR Hand washing programs AND Hand wash OR Hand washing OR Hand washings OR Handwashing OR Hand washing behaviour, OR Hand washing techniques OR Hand hygiene OR Hand disinfection OR Hand or Washings OR Hand scrubbing AND Use OR Usefulness OR Utilisation OR Benefit OR Advantages OR Effectiveness OR evaluation AND Promotion OR Sustainability Or Adoption OR Appropriateness AND Prevention OR Control OR Limit AND diarrhoea, OR dysentery OR waterborne disease OR bloody stool OR Loose stool OR Respiratory Infection OR Infection OR Cross infection 


\section{Study selection}

Identified titles from the databases were extracted and imported to Endnote X7 Reference Management System. Thereafter, duplicates were removed. The abstracts of the retained titles were retrieved and manually assessed for potential eligibility. Full articles were retrieved for the retained abstracts and these were thoroughly assessed manually for eligibility. Assessing eligibility for the articles was done independently by two reviewers using the predefined inclusion and exclusion criteria. Any disagreement between the two reviewers over the eligibility of particular studies were resolved through discussion with a third reviewer.

\section{Data collection process}

The process of data extraction started with database search of relevant articles using search terms while following the Preferred Reporting Items for Systematic Reviews and Meta-Analyses (PRISMA) [15] guidelines (see Fig. 3). A standardised form was used to extract data from the included studies for assessment of the study quality and evidence synthesis. The details included: author, year of study, type of participants, age, setting, country, sample size, study design, and methods, study purpose and objectives, intervention description, study outcome measures (see Supplementary material A). All relevant information was extracted from each article, summarised and documented (see Table 2). Two reviewers extracted data independently; discrepancies were identified and resolved through discussion with a third author. Missing data were requested from the corresponding authors of the study.

\section{Search outcome}

The search yielded a total of 4091 titles of articles of which 1696 were retained in a preliminary assessment stage after removing duplicates. Of the retained articles1623 were further excluded from the analysis because they were based on different study areas or were abstracts only. Seventy-three titles were retained, and their full articles were retrieved and assessed by two authors for eligibility. The third author validated the eligibility of the articles for inclusion in the review. From this assessment, only 20 articles met the inclusion

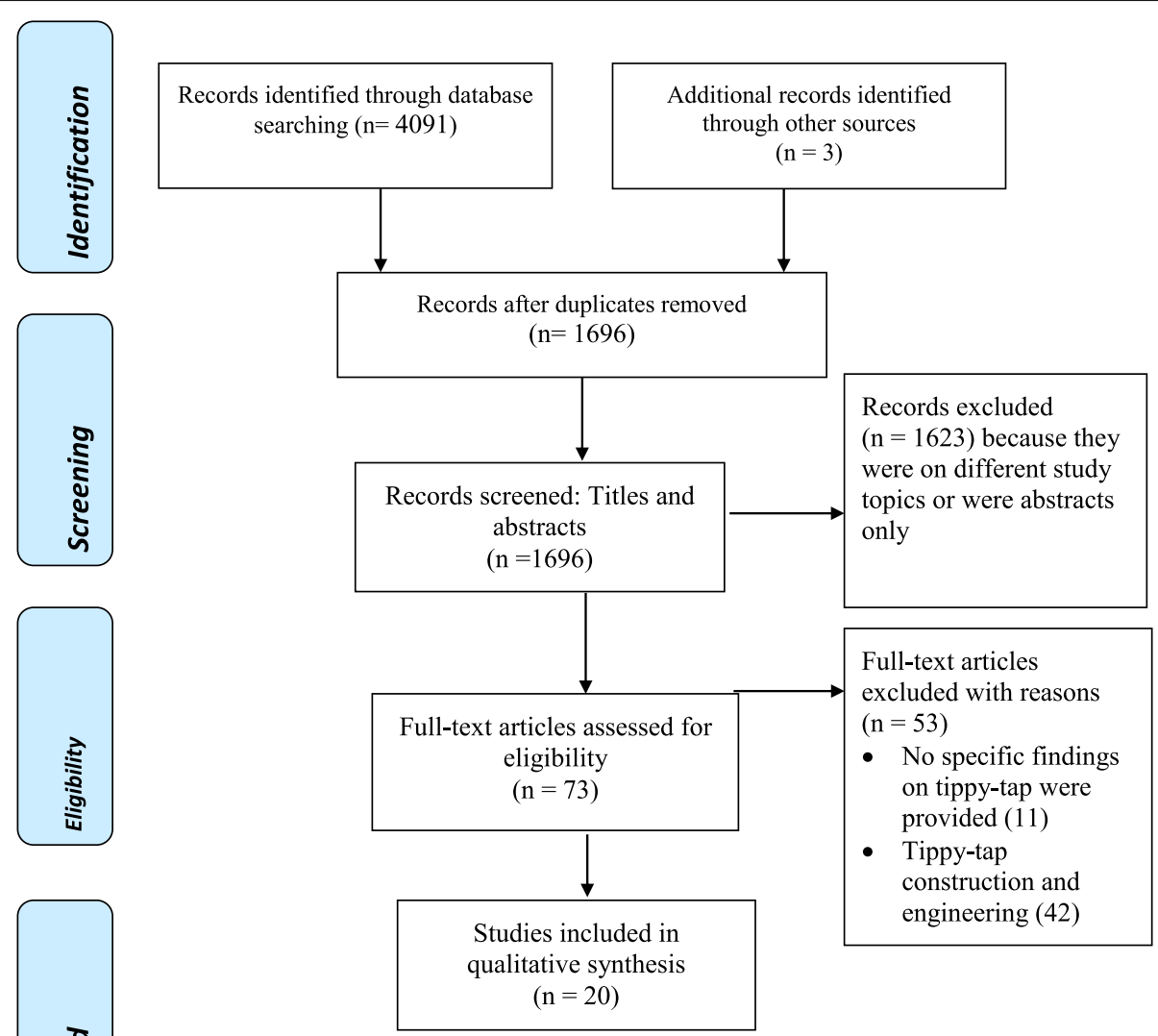

Fig. 3 PRISMA Flow Diagram [15] 


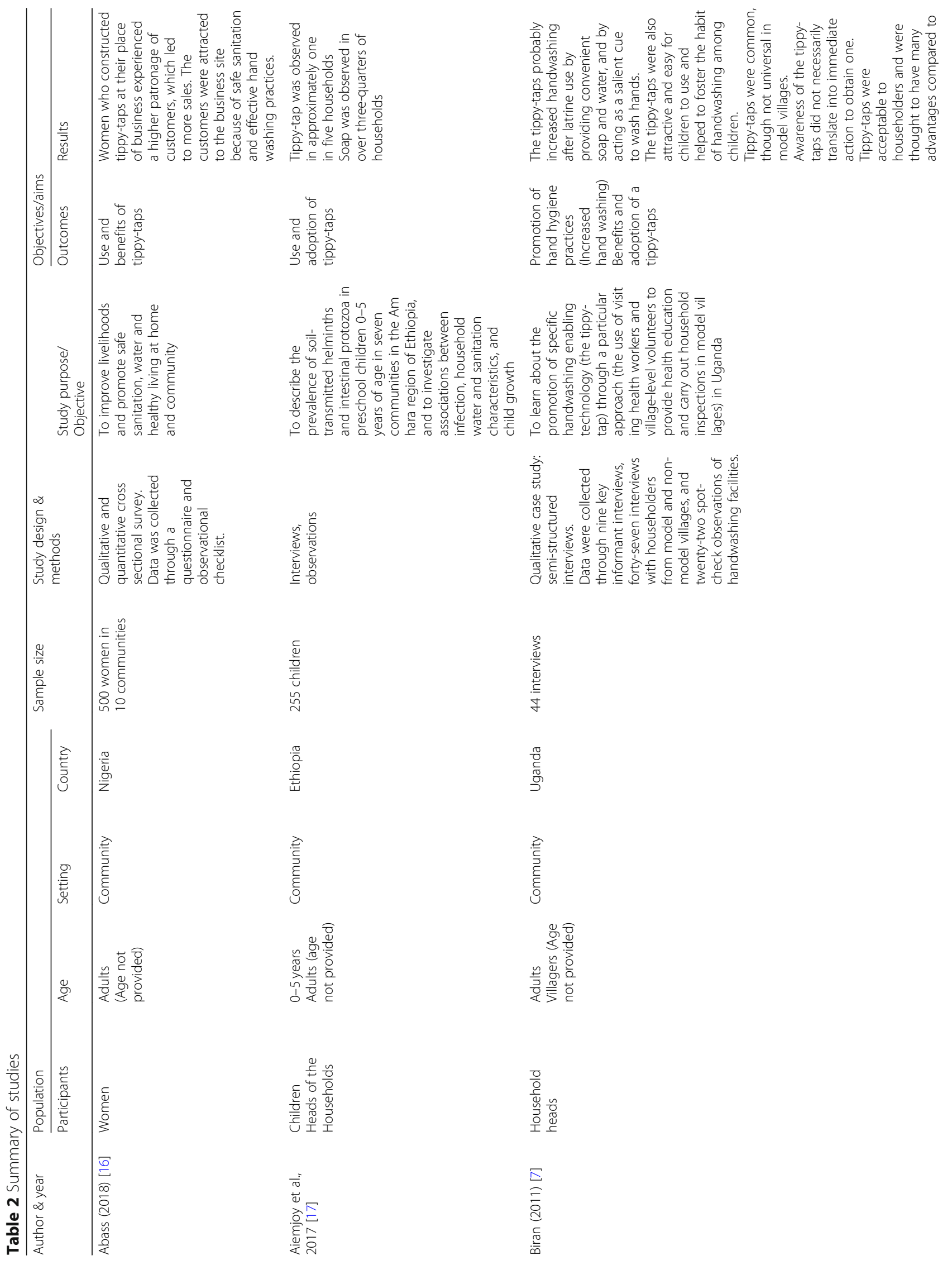




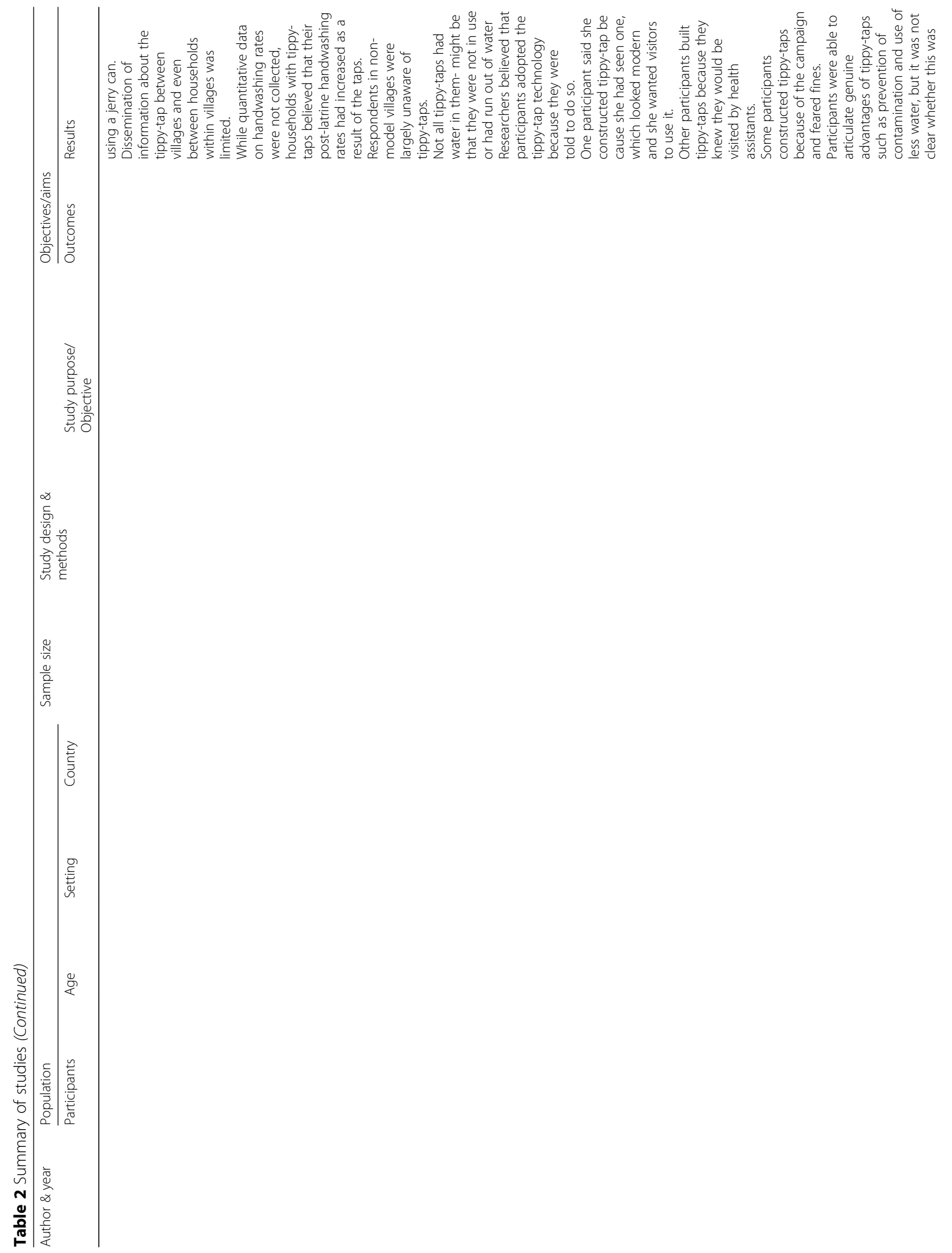




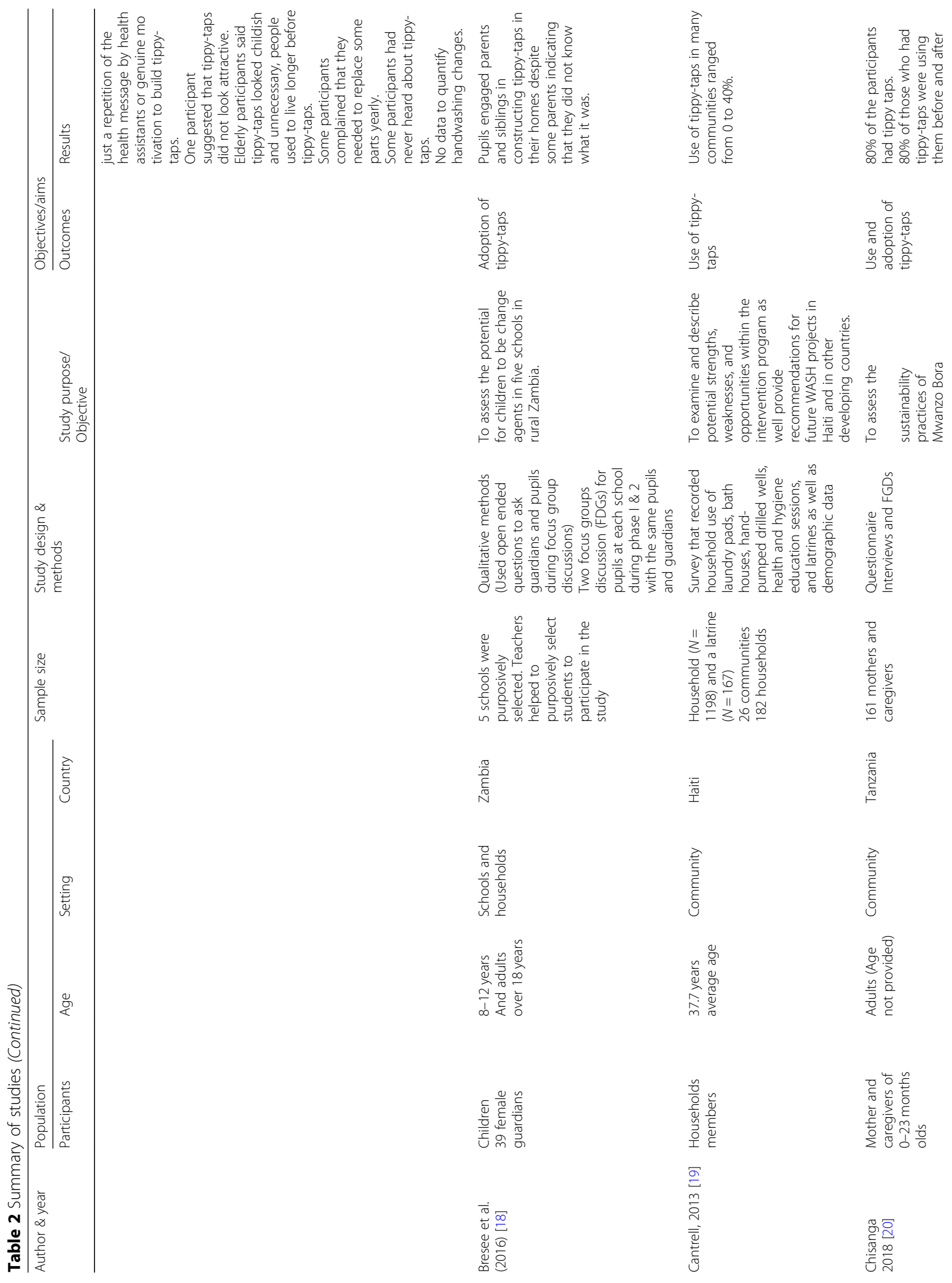




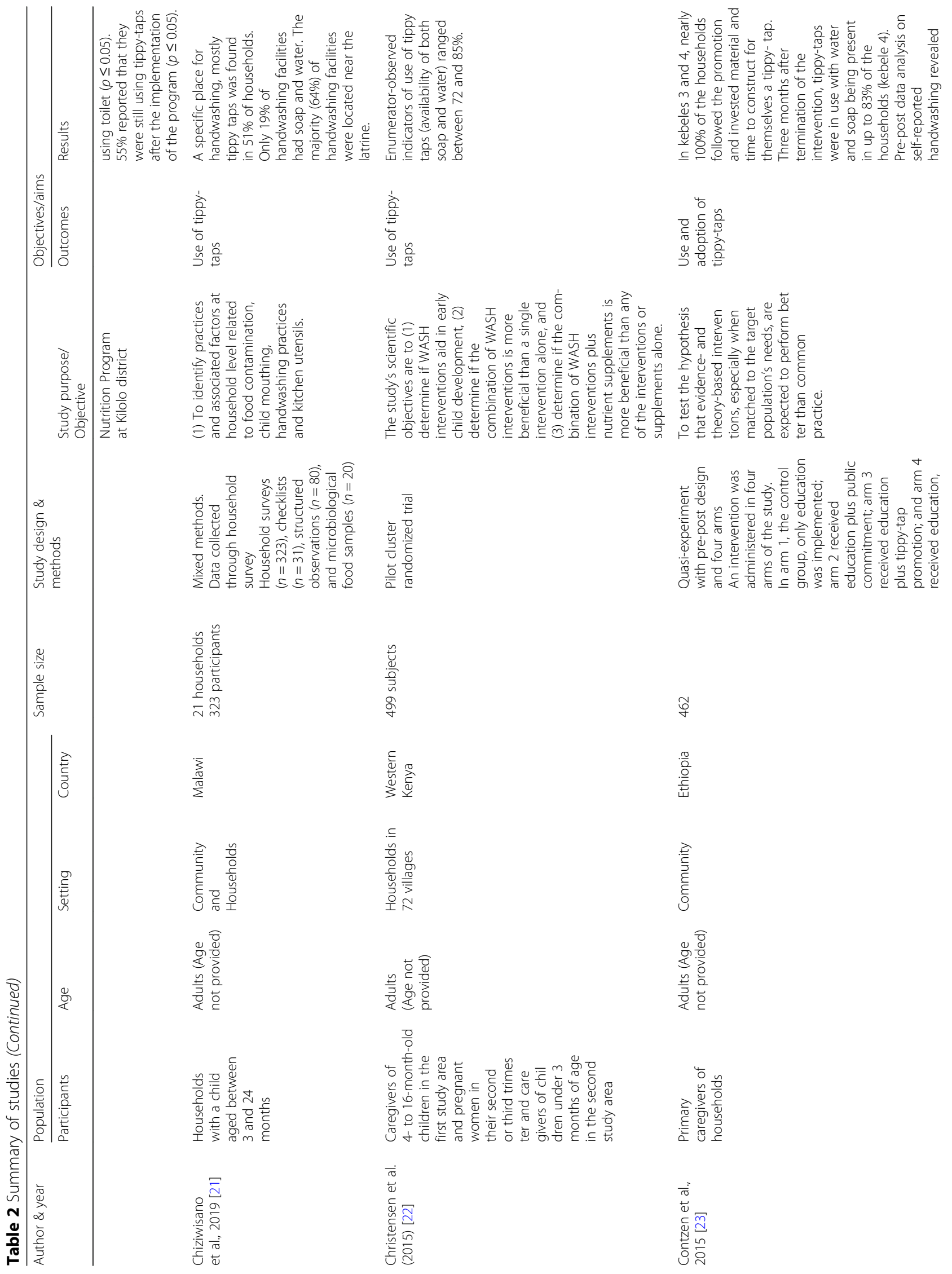




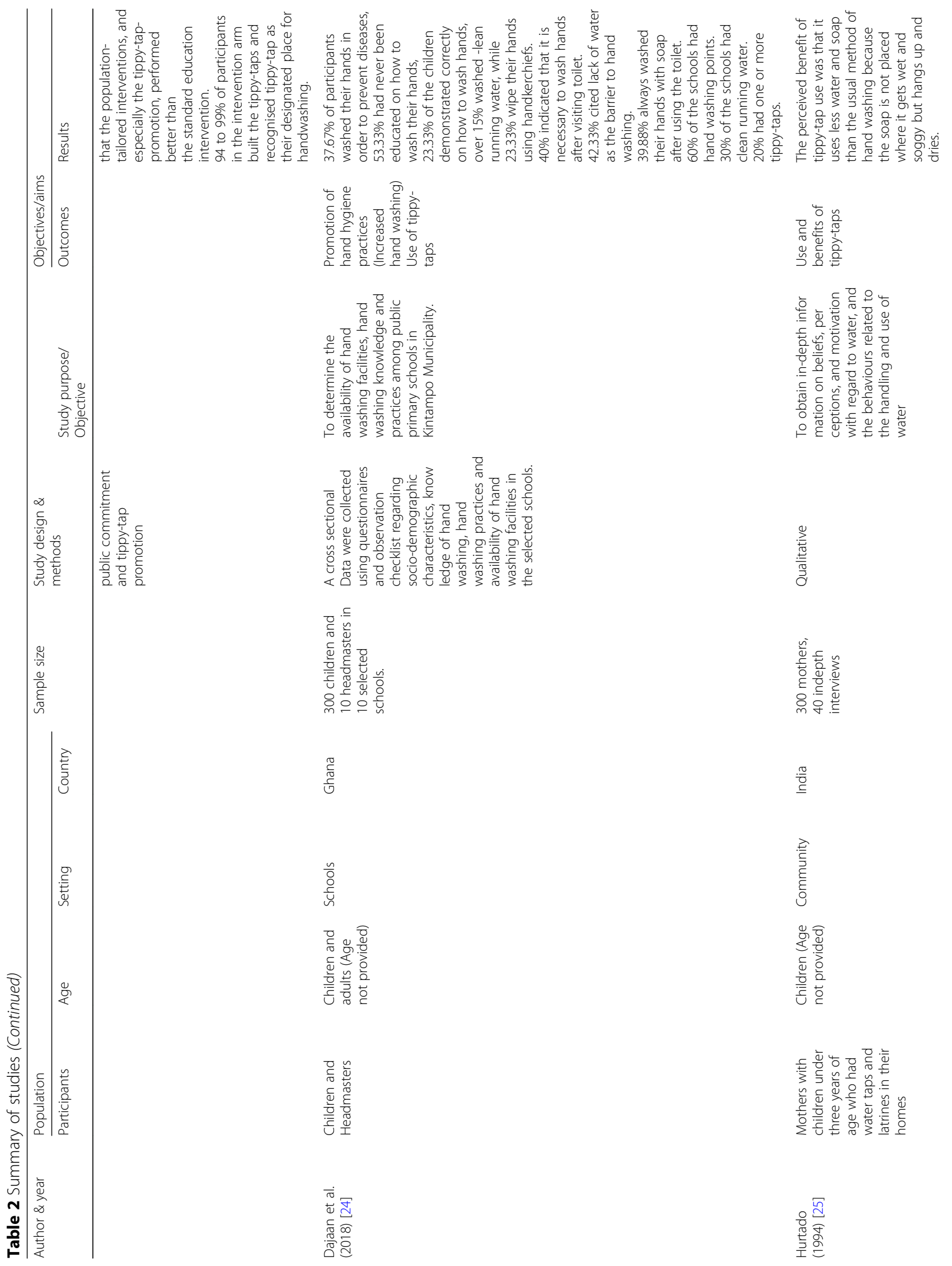




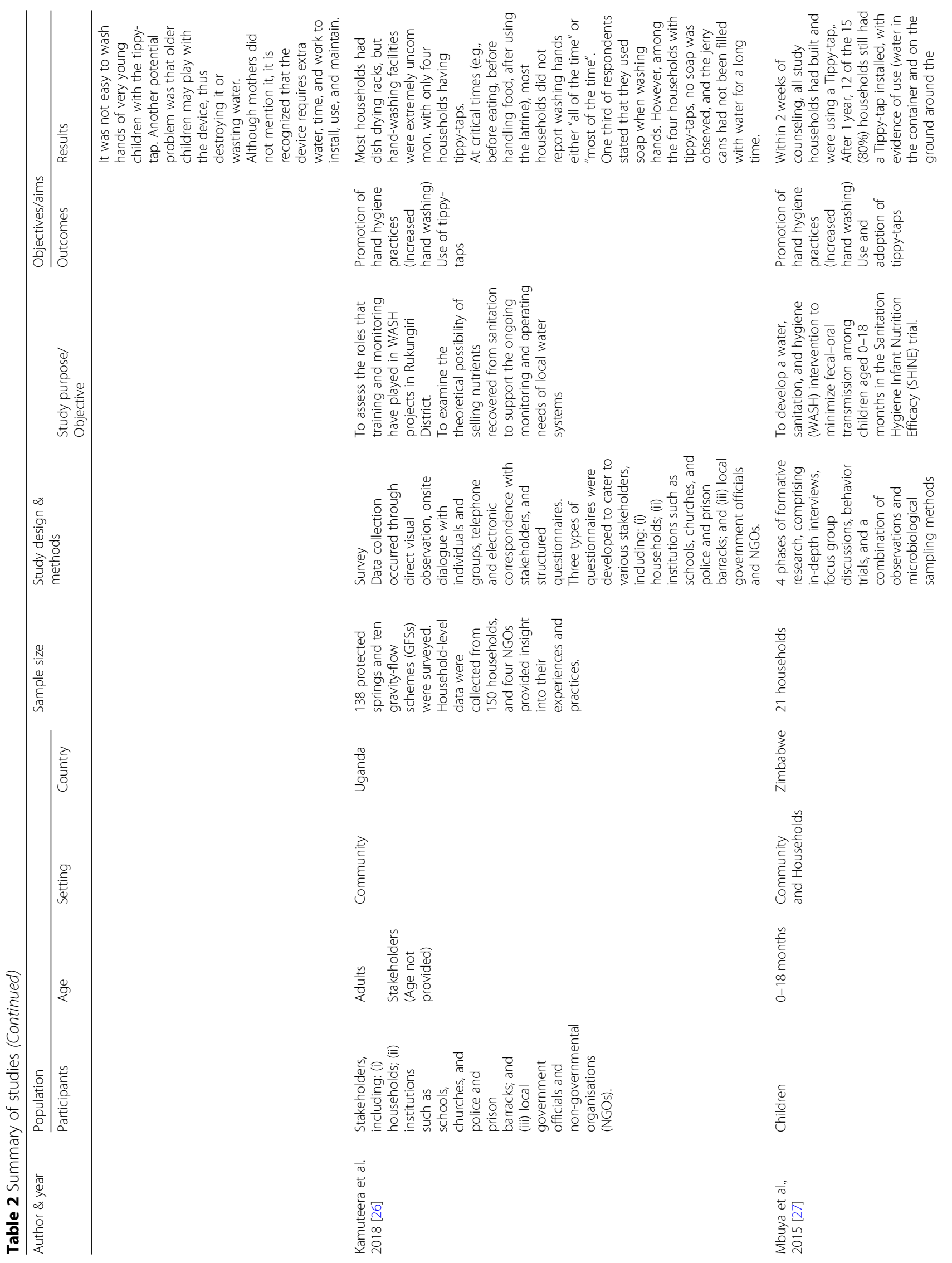




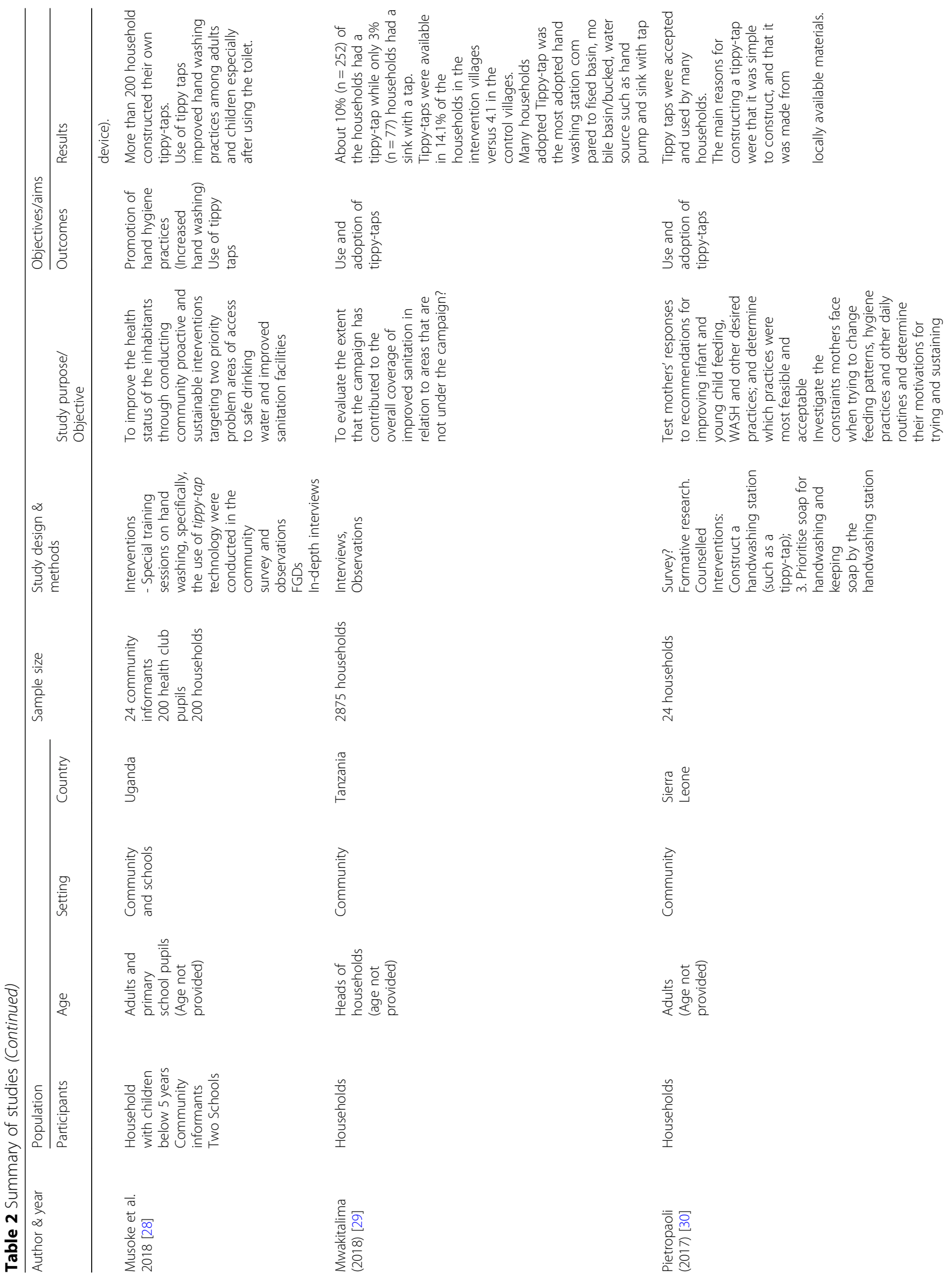




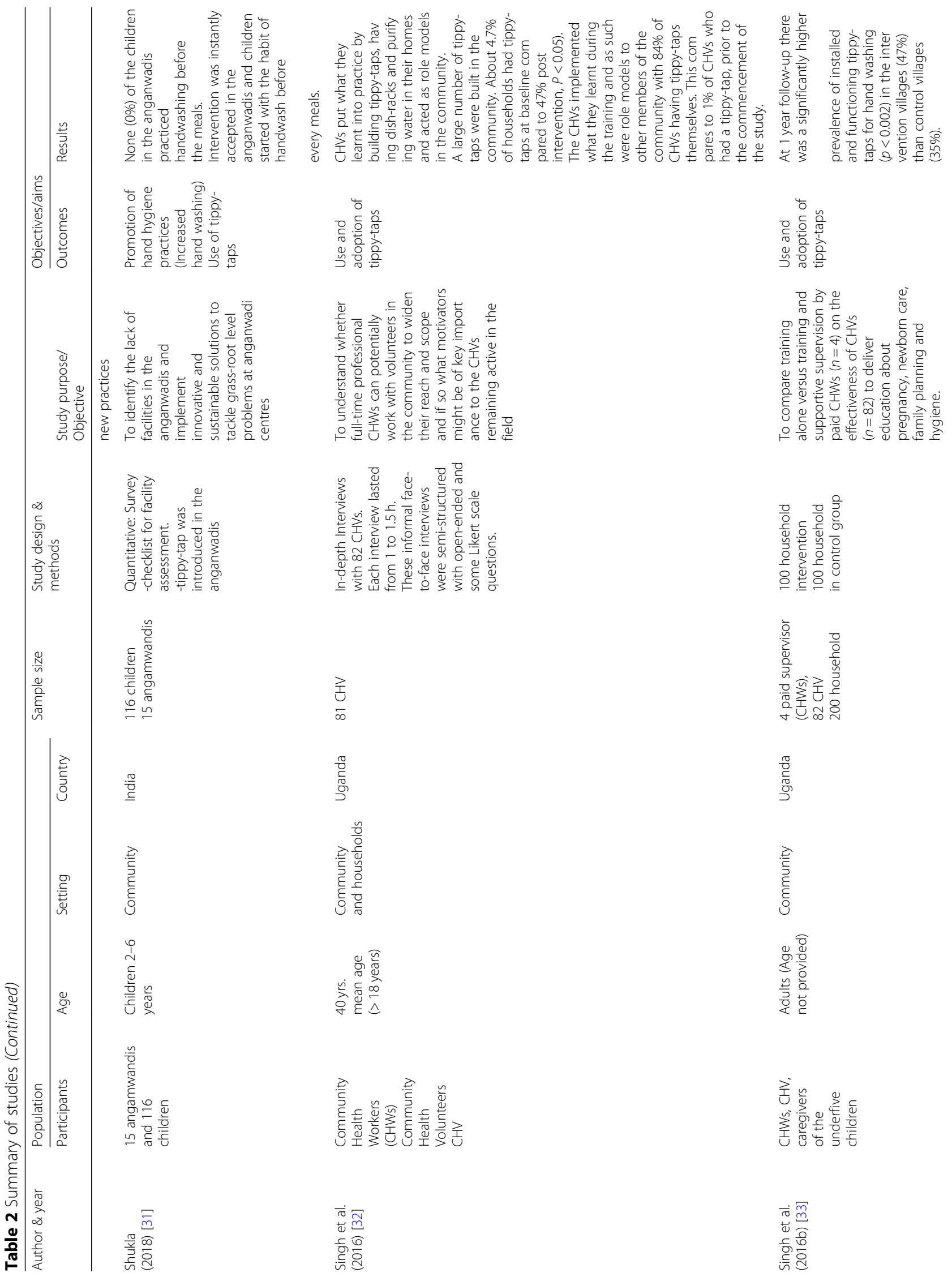




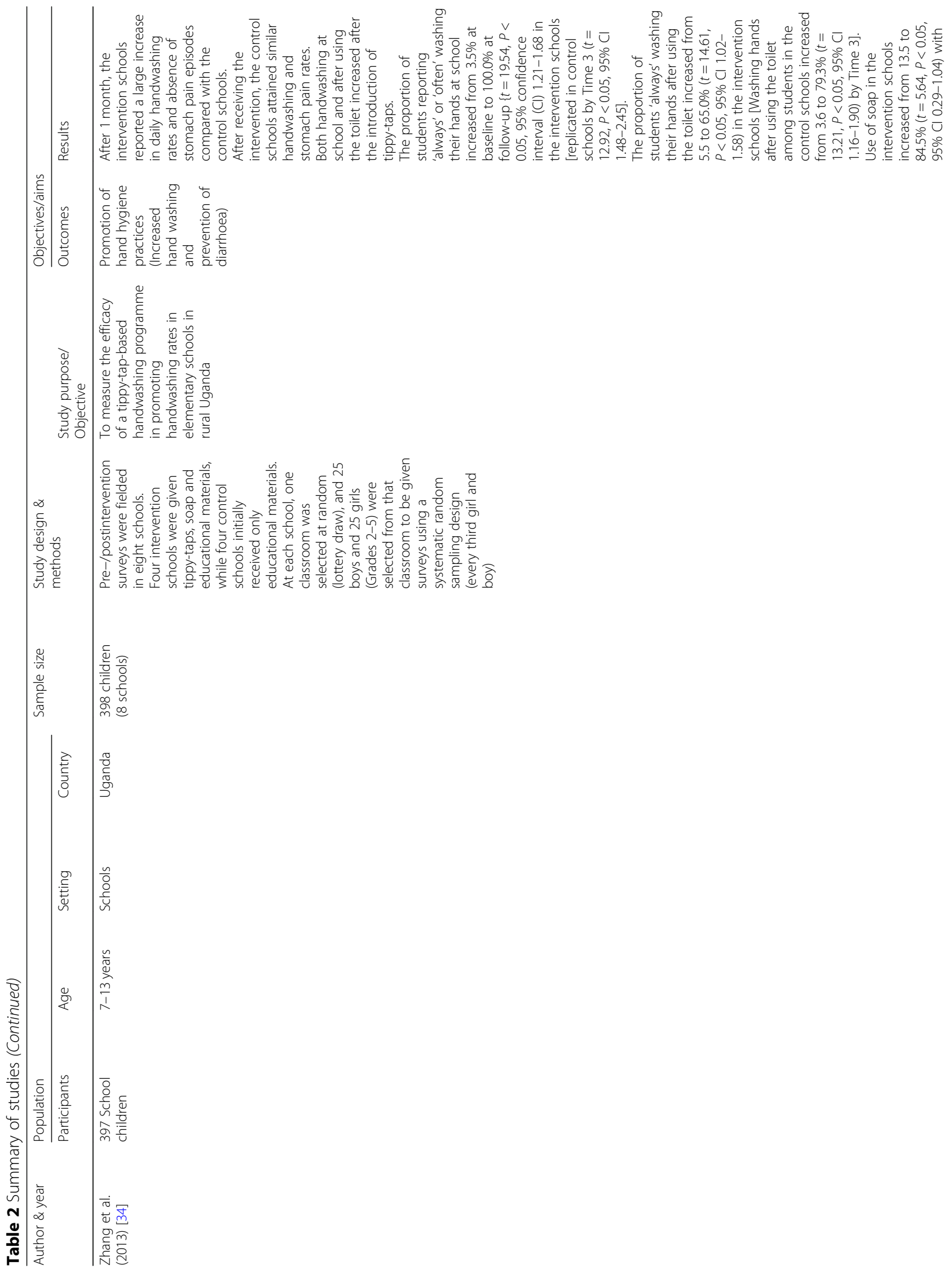




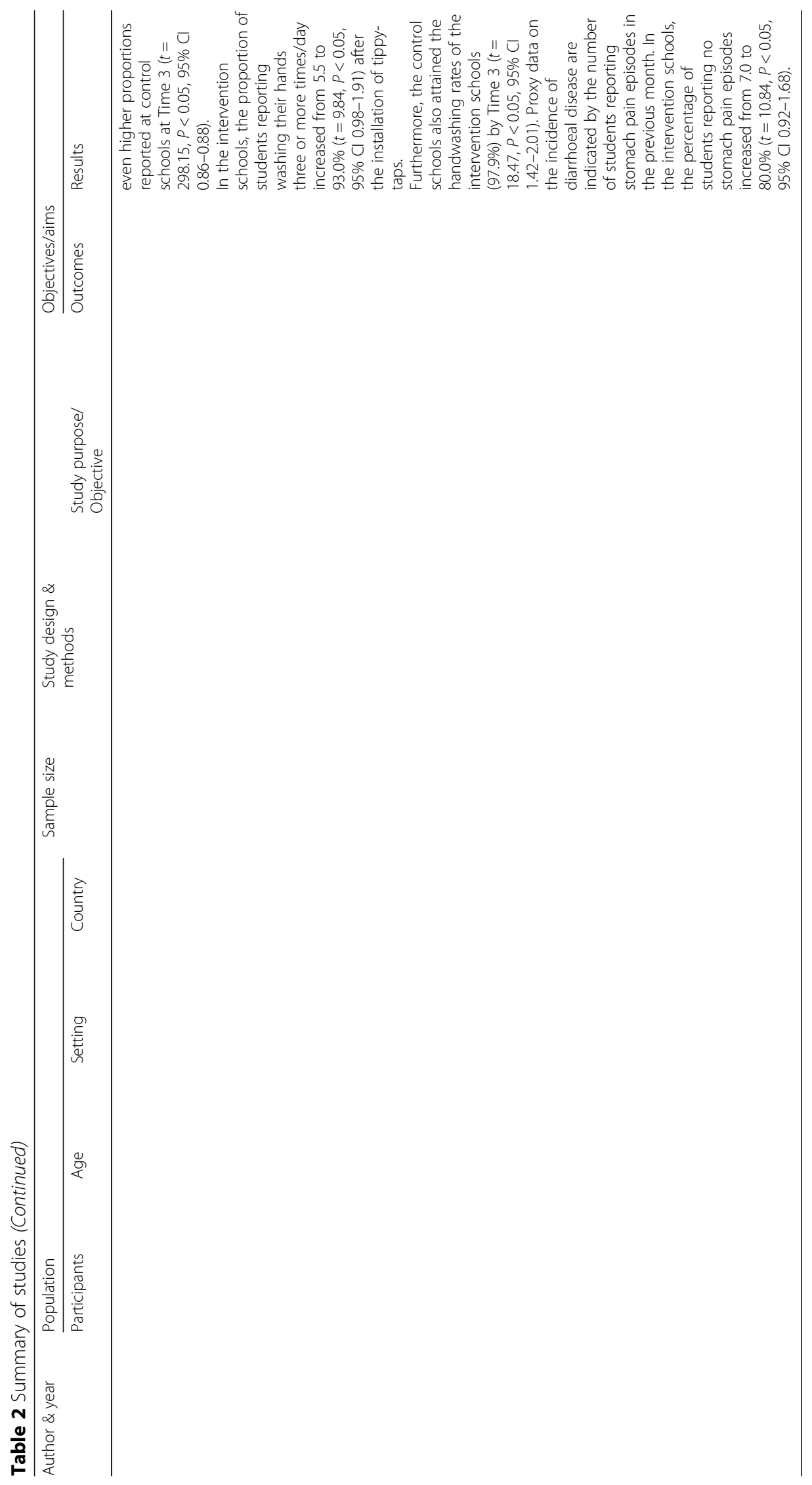


criteria. Fifty-three articles were excluded from this systematic review because they did not meet the eligibility criteria (see Fig. 3).

\section{Risk of bias/quality appraisal}

Quality of the design and reporting system were the main focus at this stage. Three review authors independently assessed the risk of bias in the included studies. The MMAT [35] was used to appraise the twenty studies included in the review critically. MMAT is a validated checklist used to appraise the quality of studies included in any systematic review with a quantitative, qualitative and mixed methods approach [36-38]. The MMAT has two general screening questions applicable to all study designs: 1) Are there clear qualitative and quantitative research questions or objectives, or is there a clear mixed-methods' question or objective? 2) Do the collected data address the research question or objective? The MMAT appraises the following study methodologies and designs: qualitative, quantitative randomised controlled, quantitative non-randomized, quantitative descriptive and mixed methods study designs. The tool is divided into five components and each component is designed to assess the quality of a specific study design. These components are qualitative, quantitative randomised controlled, quantitative non-randomized, quantitative descriptive, and mixed methods studies. All components are numbered, and each section has three to four assessment criteria. For example, assessment criteria for assessing for randomised controlled trial studies included: 1) Is there a clear description of the randomization? 2) Is there a clear description of the allocation concealment? 3) Are there complete outcome data [ $80 \%$ or above]? 4) Is there low withdrawal/dropout (below 20\%)? Each criterion equals $25 \%$ if the assessment response is 'Yes', and zero if the response is 'No'. A summation of the responses is the total score of the quality of the study in per cent and the maximum score per study is $100 \%$ (see Table 3 ). In the assessment component for mixed methods, $25 \%$ is given by default and is summed up with other scores from the criteria under this component. Overall, the higher the score, the better the quality of the study. MMAT was chosen to appraise studies in this review because it can simultaneously appraise studies of different designs, which suits different study methodologies included in this systematic review.

\section{Data synthesis}

A narrative approach was used to synthesise data. Narrative synthesis in systematic reviews is recommended when there is a great variation in variables such as outcomes, interventions, population, and methods across studies [39]. We integrated the findings from the qualitative and quantitative findings [40]. This design involves either turning qualitative data into quantitative (quantitising) or quantitative findings are turned into qualitative (qualitising) to facilitate their integration [40]. This design has been widely used in mixed methods systematic reviews $[41,42]$. We used study outcomes as themes to synthesise data. A narrative approach was also used to synthesis the quality of study and characteristics of the study characteristics.. The main category of the analysis was based on the promotion of handwashing behaviour by using tippy-tap. Under this category, the reviewers came up with three subcategories, namely: the use and benefit of tippy-tap in promoting hand hygiene; adoption of tippy-tap and its associated hand hygiene resources, and the effectiveness of tippy-tap. In this systematic review, "use" of tippy-tap refers to the situation whereby the participant merely used tippy-tap to wash hands and/or increased their handwashing during their respective project implementation. On the other hand, "adoption" of tippy-tap refers to a situation whereby the participant continued using tippy-taps even after their respective research projects or programs had stopped or constructed new tippy-taps after completion of the project. Effectiveness of tippy-tap in this study refers to proxy data of reducing infectious diseases. Content analysis was carried out to synthesise the extracted data and similar information was grouped (see Table 2). Findings were presented in narrative form as shown below. The interventions were also classified according the settings where they were implemented. The settings of the study were classified as households (peoples' houses), primary schools, and communities. Community based intervention in this study refers to interventions implemented at a public place (village level, church, and neighbourhoods). Statistical meta-analysis was not possible as the studies varied considerably on how the study outcomes were analysed by the researchers.

\section{Results \\ Quality appraisal}

Based on MMAT, nine studies scored 100\% [17-22, 24, $29,32]$. Of these, two were qualitative, five were quantitative descriptive, and one was a mixed-methods study. Nine studies scored $75 \%$ [16, 23, 25-28, 31, 33, 34] among these, three were experimental studies that had no information on blinding [23, 33, 34]; three were qualitative studies with no clear description regarding the influence of the researcher on study findings $[25,27,31]$; two were mixed methods studies that did not highlight the limitations to integration of qualitative and quantitative findings $[16,28]$; and one was a non-randomised study with a low response rate [26]. Two qualitative studies scored 50\% each because they lacked information about how data were analysed and description on whether a special consideration was given to how findings related to the 
Table 3 MMAT

\begin{tabular}{|c|c|c|c|c|}
\hline $\begin{array}{l}\text { Name of study } \\
\text { author }\end{array}$ & Type of study & Methodological quality criteria & Yes & Comments \\
\hline \multirow[t]{3}{*}{ Abass (2018) [16] } & Mixed methods & $\begin{array}{l}\text { 5.1. Is the mixed methods research design relevant to address the } \\
\text { qualitative and quantitative research questions (or objectives), or } \\
\text { the qualitative and quantitative aspects of the mixed methods } \\
\text { question (or objective)? }\end{array}$ & Y & not clear \\
\hline & & $\begin{array}{l}\text { 5.2. Is the integration of qualitative and quantitative data (or } \\
\text { results*) relevant to address the research question (objective)? }\end{array}$ & Y & \\
\hline & & $\begin{array}{l}\text { 5.3. Is appropriate consideration given to the limitations associated } \\
\text { with this integration, e.g., the divergence of qualitative and } \\
\text { quantitative data (or results*) in a triangulation design? }\end{array}$ & $\mathrm{N}$ & \\
\hline \multirow[t]{4}{*}{$\begin{array}{l}\text { Aiemjoy et al. } \\
2017[17]\end{array}$} & $\begin{array}{l}\text { Quantitative } \\
\text { cross sectional }\end{array}$ & $\begin{array}{l}\text { 4.1. Is the sampling strategy relevant to address the quantitative } \\
\text { research question (quantitative aspect of the mixed methods } \\
\text { question)? }\end{array}$ & Y & \\
\hline & & 4.2. Is the sample representative of the population understudy? & Y & \\
\hline & & $\begin{array}{l}\text { 4.3. Are measurements appropriate (clear origin, or validity known, } \\
\text { or standard instrument)? }\end{array}$ & Y & \\
\hline & & 4.4. Is there an acceptable response rate ( $60 \%$ or above)? & Y & \\
\hline Biran (2011) [7] & Qualitative & 1.1. Are the sources of qualitative data (archives, documents, & Y & Nothing on analysis \\
\hline
\end{tabular}

informants, observations) relevant to address the research question
(objective)?

1.2. Is the process for analysing qualitative data relevant to address $\mathrm{N}$ the research question (objective)?

1.3. Is appropriate consideration given to how findings relate to $\quad Y$ the context, e.g., the setting, in which the data were collected?

1.4. Is appropriate consideration given to how findings relate to $\mathrm{N}$ researchers' influence, e.g., through their interactions with participants?

Breese et al., Qualitative 1.1. Are the sources of qualitative data (archives, documents, $\quad$ Y (2016) informants, observations) relevant to address the research question (objective)?

1.2. Is the process for analyzing qualitative data relevant to address $Y$ the research question (objective)?

1.3. Is appropriate consideration given to how findings relate to $Y$ the context, e.g., the setting, in which the data were collected?

1.4. Is appropriate consideration given to how findings relate to $Y$ researchers' influence, e.g., through their interactions with participants?

Cantrell,

(2013) [19]

Chisanga et al. 2018 [20]
Quantitative descriptive Survey

Quantitative cross sectional

Mixed methods

4.1. Is the sampling strategy relevant to address the quantitative research question (quantitative aspect of the mixed methods question)?

4.2. Is the sample representative of the population understudy? $\quad Y$

4.3. Are measurements appropriate (clear origin, or validity known, $Y$ or standard instrument)?

4.4. Is there an acceptable response rate ( $60 \%$ or above)? applicable, an acceptable response rate (60\% or above), or an acceptable

4.1. Is the sampling strategy relevant to address the quantitative research question (quantitative aspect of the mixed methods question)?

4.2. Is the sample representative of the population understudy? $\quad Y$

4.3. Are measurements appropriate (clear origin, or validity known, $Y$ or standard instrument)?

4.4. Is there an acceptable response rate ( $60 \%$ or above)? 
Table 3 MMAT (Continued)

\begin{tabular}{|c|c|c|c|c|}
\hline $\begin{array}{l}\text { Name of study } \\
\text { author }\end{array}$ & Type of study & Methodological quality criteria & Yes Comments & Score \\
\hline & & $\begin{array}{l}\text { the qualitative and quantitative aspects of the mixed methods } \\
\text { question (or objective)? }\end{array}$ & & \\
\hline & & $\begin{array}{l}\text { 5.2. Is the integration of qualitative and quantitative data (or } \\
\text { results*) relevant to address the research question (objective)? }\end{array}$ & Y & \\
\hline & & $\begin{array}{l}\text { 5.3. Is appropriate consideration given to the limitations associated } \\
\text { with this integration, e.g., the divergence of qualitative and } \\
\text { quantitative data (or results*) in a triangulation design? }\end{array}$ & Y & \\
\hline \multirow[t]{4}{*}{$\begin{array}{l}\text { Christensen et al. } \\
\text { (2015) [22] }\end{array}$} & $\begin{array}{l}\text { Randomized } \\
\text { controlled trial }\end{array}$ & $\begin{array}{l}\text { 2.1. Is there a clear description of the randomization (or an } \\
\text { appropriate sequence generation)? }\end{array}$ & Y & $100 \%$ \\
\hline & & $\begin{array}{l}\text { 2.2. Is there a clear description of the allocation concealment (or } \\
\text { blinding when applicable)? }\end{array}$ & Y & \\
\hline & & 2.3. Are there complete outcome data ( $80 \%$ or above)? & Y & \\
\hline & & 2.4. Is there low withdrawal/drop-out (below 20\%)? & Y & \\
\hline \multirow[t]{4}{*}{$\begin{array}{l}\text { Contzen et al. } \\
\text { (2015) [23] }\end{array}$} & Quasi-experiment & $\begin{array}{l}\text { 2.1. Is there a clear description of the randomization (or an } \\
\text { appropriate sequence generation)? }\end{array}$ & Y & $75 \%$ \\
\hline & & $\begin{array}{l}\text { 2.2. Is there a clear description of the allocation concealment (or } \\
\text { blinding when applicable)? }\end{array}$ & $\mathrm{N}$ & \\
\hline & & 2.3. Are there complete outcome data ( $80 \%$ or above)? & Y & \\
\hline & & 2.4. Is there low withdrawal/drop-out (below 20\%)? & Y & \\
\hline \multirow[t]{4}{*}{$\begin{array}{l}\text { Dajaan et al. } \\
\text { (2018) [24] }\end{array}$} & $\begin{array}{l}\text { Quantitative } \\
\text { cross sectional }\end{array}$ & $\begin{array}{l}\text { 4.1. Is the sampling strategy relevant to address the quantitative } \\
\text { research question (quantitative aspect of the mixed methods } \\
\text { question)? }\end{array}$ & Y & $100 \%$ \\
\hline & & 4.2. Is the sample representative of the population understudy? & Y & \\
\hline & & $\begin{array}{l}\text { 4.3. Are measurements appropriate (clear origin, or validity known, } \\
\text { or standard instrument)? }\end{array}$ & Y & \\
\hline & & $\begin{array}{l}\text { 4.4. Is there an acceptable response rate ( } 60 \% \text { or above)? } \\
\text { applicable, an acceptable response rate ( } 60 \% \text { or above), or an } \\
\text { acceptable }\end{array}$ & Y & \\
\hline Hurtado & Qualitative & 1. Are the sources of qualitative data (archives, documents, & not clear & $75 \%$ \\
\hline
\end{tabular}

1. Are the sources of qualitative data (archives, documents, (objective)?

1.2. Is the process for analysing qualitative data relevant to address $Y$ the research question (objective)?

1.3. Is appropriate consideration given to how findings relate to $\quad Y$ the context, e.g., the setting, in which the data were collected?

1.4. Is appropriate consideration given to how findings relate to N researchers' influence, e.g., through their interactions with participants?

Kamuteera et al. Quantitative cross 2018 [26] sectional survey
Mbuya et al., Qualitative (2015) [27]
4.1. Is the sampling strategy relevant to address the quantitative research question (quantitative aspect of the mixed methods question)?

4.2. Is the sample representative of the population understudy? $\quad \mathrm{Y}$

4.3. Are measurements appropriate (clear origin, or validity known, $Y$ or standard instrument)?

4.4. Is there an acceptable response rate ( $60 \%$ or above)? applicable, an acceptable response rate (60\% or above), or an acceptable

1.1. Are the sources of qualitative data (archives, documents, informants, observations) relevant to address the research question (objective)?

1.2. Is the process for analyzing qualitative data relevant to address $Y$ the research question (objective)?

1.3. Is appropriate consideration given to how findings relate to $\quad Y$ 
Table 3 MMAT (Continued)

\begin{tabular}{|c|c|c|c|c|}
\hline $\begin{array}{l}\text { Name of study } \\
\text { author }\end{array}$ & Type of study & Methodological quality criteria & Yes & Comments \\
\hline & & the context, e.g., the setting, in which the data were collected? & & \\
\hline & & $\begin{array}{l}\text { 1.4. Is appropriate consideration given to how findings relate to } \\
\text { researchers' influence, e.g., through their interactions with } \\
\text { participants? }\end{array}$ & $\mathrm{N}$ & \\
\hline \multirow[t]{3}{*}{$\begin{array}{l}\text { Musoke et all. } \\
2018[28]\end{array}$} & Mixed methods & $\begin{array}{l}\text { 5.1. Is the mixed methods research design relevant to address the } \\
\text { qualitative and quantitative research questions (or objectives), or } \\
\text { the qualitative and quantitative aspects of the mixed methods } \\
\text { question (or objective)? }\end{array}$ & Y & $\begin{array}{l}\text { Superficial analysis } \\
\text { procedures reported }\end{array}$ \\
\hline & & $\begin{array}{l}\text { 5.2. Is the integration of qualitative and quantitative data (or } \\
\text { results*) relevant to address the research question (objective)? }\end{array}$ & Y & \\
\hline & & $\begin{array}{l}\text { 5.3. Is appropriate consideration given to the limitations associated } \\
\text { with this integration, e.g., the divergence of qualitative and } \\
\text { quantitative data (or results*) in a triangulation design? }\end{array}$ & $\mathrm{N}$ & \\
\hline Mwakitalima & Quantitative cross & 3.1. Are participants (organizations) recruited in a way that & Y & 5KM apart \\
\hline
\end{tabular}

(2018) [29]

Pietropaoli (2017) [30]

Shukla (2018)

[31]

Singh et al. (2016) [32]

Singh et al. (2016b) [33]

\section{sectional}

Qualitative

Quantitative descriptive

Qualitative

Randomised controlled trial
3.1. Are participants (organiz
minimizes selection bias?

3.2. Are measurements appropriate (clear origin, or validity known, Y or standard instrument; and absence of contamination between groups when appropriate) regarding the exposure/intervention and outcomes?

3.3. In the groups being compared (exposed vs. non-exposed; with $Y$ intervention vs. without; cases vs. controls), are the participants comparable, or do researchers take into account (control for) the difference between these groups?

3.4. Are there complete outcome data (80\% or above), and, when $Y$ applicable, an acceptable response rate (60\% or above), or an acceptable follow-up rate for cohort studies (depending on the duration of follow-up)?

1.1. Are the sources of qualitative data (archives, documents, informants, observations) relevant to address the research question (objective)?

1.2. Is the process for analyzing qualitative data relevant to address $\mathrm{N}$ the research question (objective)?

1.3. Is appropriate consideration given to how findings relate to the context, e.g., the setting, in which the data were collected?

1.4. Is appropriate consideration given to how findings relate to researchers' influence, e.g., through their interactions with participants?

4.1. Is the sampling strategy relevant to address the quantitative research question (quantitative aspect of the mixed methods question)?

4.2. Is the sample representative of the population understudy?

4.3. Are measurements appropriate (clear origin, or validity known, $Y$ or standard instrument)?

4.4. Is there an acceptable response rate (60\% or above)?

1.1. Are the sources of qualitative data (archives, documents, $\quad Y$ informants, observations) relevant to address the research question (objective)?

1.2. Is the process for analysing qualitative data relevant to address $Y$ the research question (objective)?

1.3. Is appropriate consideration given to how findings relate to the context, e.g., the setting, in which the data were collected?

1.4. Is appropriate consideration given to how findings relate to $Y$ researchers' influence, e.g., through their interactions with participants?

2.1. Is there a clear description of the randomization (or an appropriate sequence generation)?

Y Nothing on analysis

$50 \%$

Y Info not given 
Table 3 MMAT (Continued)

\begin{tabular}{|c|c|c|c|c|}
\hline $\begin{array}{l}\text { Name of study } \\
\text { author }\end{array}$ & Type of study & Methodological quality criteria & Yes Comments & Score \\
\hline & & $\begin{array}{l}\text { 2.2. Is there a clear description of the allocation concealment (or } \\
\text { blinding when applicable)? }\end{array}$ & $\mathrm{N}$ & \\
\hline & & 2.3. Are there complete outcome data ( $80 \%$ or above)? & Y & \\
\hline & & 2.4. Is there low withdrawal/drop-out (below 20\%)? & Y & \\
\hline \multirow[t]{4}{*}{$\begin{array}{l}\text { Zhang et al. } \\
\text { (2013) [34] }\end{array}$} & $\begin{array}{l}\text { Randomized } \\
\text { controlled trial }\end{array}$ & $\begin{array}{l}\text { 2.1. Is there a clear description of the randomization (or an } \\
\text { appropriate sequence generation)? }\end{array}$ & Y & $75 \%$ \\
\hline & & $\begin{array}{l}\text { 2.2. Is there a clear description of the allocation concealment (or } \\
\text { blinding when applicable)? }\end{array}$ & $\mathrm{N}$ & \\
\hline & & 2.3. Are there complete outcome data ( $80 \%$ or above)? & Y & \\
\hline & & 2.4. Is there low withdrawal/drop-out (below 20\%)? & Y & \\
\hline
\end{tabular}

*Both qualitative and quantitative results

researcher's influence $[7,30]$. MMAT has no cut-off point for the quality of studies, but we considered 'less than $50 \%$ 'score as low quality. However, none of our selected studies scored below 50\%. With an average MMAT score of $82.5 \%$ across the included studies, the studies are considered to be of high quality.

\section{Study characteristics}

Twenty studies met the eligible criteria. Of these, six were conducted in Uganda [7, 26, 28, 32-34], two in Ethiopia [17, 23], and two in Tanzania [20, 29]. Furthermore, one study was conducted in each of the following countries: Zambia [18], Zimbabwe [27], Kenya [22], Nigeria [16], Haiti [19], Malawi [21], Ghana [24] and Sierra Leone ([30] (See Table 2). In terms of study design, six qualitative studies $[7,18,25,27,30,32], 11$ quantitative studies $[17,19,20,22-24,26,29,31,32,34]$, and three mixed methods studies $[16,21,28]$ were evaluated. Data collection in the qualitative studies was through focus group interviews, semi-structured questionnaire and in-depth interviews. The quantitative studies utilised quasi-experiment, pre-post survey, cross-sectional survey and cluster randomised trials study approaches (see Table 2).

A total of 11 studies were conducted in the community $[7,16,17,19-23,25-30]$ and four were conducted in schools $[18,24,28,34]$. The study population in six studies were children while 16 studies were conducted with adults. The youngest participants were infants less than 8 months old [27] and the oldest was 40 years [32] The number of participants in each study varied from 21 [18] to 2875 [29].

\section{Summary of the findings}

Studies included in this review were analysed based on the following three outcomes: the use and benefit of tippy-tap in promoting hand hygiene; adoption of tippytap and its associated hand hygiene resources, and the effectiveness of tippy-tap. These sub-categories were generated from the objective of the study. The presentation and interpretation of the results follow these categories as narrated below.

Use and benefits of tippy-tap in promoting hand hygiene The use of tippy-taps for handwashing among household members or school children was reported by authors of 16 studies conducted in Nigeria, Haiti, Malawi, Ghana, India, Tanzania, Uganda, Sierra Leone, Kenya and Ethiopia [7, 16, 17, 19-26, 28-31, 34]. The use of tippytap among the participants in the 16 studies ranged from $2.7 \%$ [26] to $80 \%$ [20].

Concerning the benefits of using tippy-taps, authors of three studies $[7,23,34]$ reported an increase in handwashing practice by participants after being exposed to tippy-tap. In a randomised controlled trial in Uganda four intervention and four control schools were recruited into the study [34]. At each school, one classroom was selected randomly (lottery draw), and 25 boys and 25 girls (Grades 2-5) were selected from that classroom using a systematic random sampling design (every third girl and boy). Data were collected at three waves of 1 month apart intervals. The first wave was a baseline survey that was followed by the provision of soap and handwashing education to four intervention schools. The second wave was followed by the introduction of tippy-taps and provision of soap to the intervention group. Lastly, the post-intervention survey was carried out at the last wave. The four control schools received health education only through-out the experiment and were provided with tippy-taps post-study interventions. The researchers reported an increased estimate in the proportion of students reporting 'always' or 'often' washing their hands at school from $3.5 \%$ at baseline to $100.0 \%$ at follow-up $(t=19.54, P<0.05$, 95\% CI $1.21-$ 1.68) in the intervention schools. When the similar intervention was replicated in the control schools by Time 3, there was an increase in handwashing $(t=12.92$, $P<0.05,95 \%$ CI 1.48-2.45] [34]. In the same study, it 
was observed that the proportion of students 'always' washing their hands after using the toilet increased from 5.5 to $65.0 \%(t=14.61, P<0.05,95 \%$ CI $1.02-1.58)$ in the intervention schools, while in the control schools it only increased from 3.6 to $79.3 \%(t=13.21, P<0.05$, 95\% CI 1.16-1.90) by Time 3 when the same intervention was replicated [34].

In addition, compared to control schools, introduction of tippy-taps increased the use of soap by students in the intervention schools in an experiential study from 13.5 to $84.5 \%(t=5.64, P<0.05,95 \%$ CI $0.29-1.04)$; handwashing from 5.5 to $93.0 \%(t=9.84, P<0.05,95 \%$ CI $0.98-1.91$ ) and handwashing after using the toilet from 5.5 to $65.0 \%(t=14.61, P<0.05,95 \%$ CI $1.02-1.58)$ [34]. Similarly, another study [7] found that tippy-taps increased handwashing after latrine use by providing convenient soap and water, and by acting as a salient cue to handwashing. Although quantitative data on handwashing rates were not collected, participants in households with tippy-taps believed that their post-latrine handwashing rates had increased as a result of the tippy-taps [7]. Pre- and post-data analysis on self-reported handwashing revealed that the population-tailored interventions, especially the tippy-tap-promotion, performed better than the standard education intervention (education intervention, the f-diagram exercise, an often applied intervention tool) [18]. In a study conducted by Christensen and colleagues [22], the use of tippy-tap was measured through the availability of handwashing resources (soap and water) at the tippy-tap station. These researchers found that enumerator-observed indicators of use were still high (72-85\% for having both soap and water present at the tippy-tap station) [22]. In an Indian qualitative study, most participants reported using tippytap because of its benefits [25]. The participants reported that handwashing using tippy-tap requires less water and soap compared to the usual method of handwashing [25]. However, in the same study [25] participants indicated the following as challenges of the tippytap handwashing technology: it was not easy to wash hands of very young children with the tippy-tap; there was a potential problem that older children may play with the device, thus destroying it or wasting water; it was also recognized that the device required extra water, time, and work to install, use, and maintain. In addition, a study by Biran [7], one participant suggested that tippy-taps did not look attractive, elderly participants said tippy-taps looked childish and unnecessary, and that people used to live longer even before tippy-taps were developed.

On the other hand, the economic benefits of tippytaps were reported by the authors of a Nigerian study [16]. The installation of tippy-taps in small scale business facilities by women who were involved in selling food items led to an increase in the number of customers, which resulted in more sales and profits.

\section{Adoption of tippy-tap and its associated hand hygiene resources}

Authors of six studies assessed the adoption of tippytaps by households $[7,18,22,23,32,33]$. In a study conducted by Christensen [22], the intervention households were significantly more likely to have a place for handwashing (71-85 percentage point increases) with soap available (49-66 percentage point increases) than controls. These authors also noted an increase of $86 \%$ in having a dedicated location for tippy-taps. Similarly, in another study, teachers educated school going children on tippy-tap as a handwashing station [18]. Although these children were not directly asked to construct tippy-tap, they all managed to attempt building one or influence their parents to assist them. Their parents trusted the information received from their children. The tippy-taps were also found to be attractive, easy to use and helpful in fostering the habit of handwashing among children [18].

Signh et al. [33] engaged the community in a hand hygiene promotion program. At 1 year follow-up, the researchers noted a $47 \%$ installation of functioning tippytaps in the intervention villages compared to $35 \%$ in the control villages $(p<0.002)$ [33]. There was a significant increase in tippy-tap installation by community members from $4.7 \%$ of households at baseline to $47 \%$ of homes after the intervention, following the demonstrations to construct the device by community health volunteers (CHVs). The CHVs were trained on the tippy tap construction and acted as role models to other community members. Furthermore, there was a great improvement in owning tippy-taps by $\mathrm{CHVs}$ from $1 \%$ at baseline to $84 \%$ after interventions [33]. Another significant evidence of adoption of tippy-taps was observed in a study where all study households built tippy-taps within 2 weeks of counselling [27]. After 1 year of tippytap promotion, $80 \%$ of the households still had a tippytap installed, with evidence of use (water in the container and on the ground around the device). Similar results were observed in a study by Contzen and colleagues [23] in which, close to $100 \%$ of the households followed the promotion and invested material and time to construct their tippy-tap. In the same study, all participants in the intervention group constructed tippytaps and about $83 \%$ of these were still operational 3 months after termination of the interventions.

Although there is limited awareness on tippy-tap, having knowledge about tippy-tap did not result in immediate construction of the station $[7,18]$. The researcher thought that study participants constructed a tippy-taps because they were asked to do so, or they anticipated 
that the researcher would be visiting them regularly to evaluate the adoption of the technology [7]. Some participants constructed tippy-tap as a result of campaigns and fear of fines from community leaders [7].

\section{Effectiveness of tippy-tap}

Out of twenty articles under review, only one study [34] had an incidence of diarrhoea as an outcome measure. The study was conducted in a school setting in Uganda and aimed at measuring the efficacy of a tippy-tap-based handwashing programme in promoting handwashing rates in elementary schools in rural Uganda. Zhang and colleagues [34] used the pre-and post-intervention surveys in which four intervention schools were given tippy-taps, soap and educational materials, while four control schools initially received only educational materials. Proxy data for assessing the effectiveness of tippytaps in reducing diarrhoeal disease was indicated by the number of students reporting stomach pain episodes in the previous month. The authors of the study found that in the intervention schools, the percentage of students reporting no stomach pain episodes increased from $7 \%$ at baseline to $80 \%$ after the intervention $(t=10.84, P<$ $0.05,95 \%$ CI $0.92-1.68$ ) [34]. However, no proxy data was provided on the trend of diarrhoea in the control group.

\section{Discussion}

The aim of this systematic review was to assess the use, benefits, adoption and effectiveness of tippy-tap handwashing station in resource-limited settings. A total of twenty articles were identified and reviewed. The findings of our systematic review show that the availability of tippy-taps increased handwashing and use of soap among participants. Furthermore, the majority of people who were oriented to tippy-taps or recruited to tippytap studies built their tippy-tap stations even after the end of promotional activities or programs. In one study, tippy-taps were found to be effective in preventing stomach pain episodes among participants [34].

There is sufficient evidence that hand washing is a single most important intervention for preventing diarrhoeal and respiratory infections, yet the rate of handwashing in resource-limited settings is very low [3, 43-45]. Indeed, with frequent global outbreaks of infectious diseases such as COVID-19, and Ebola, the importance of identifying a cost-effective hand handwashing enabling technologies cannot be overemphasized. The findings of this review suggest that tippy-taps have a great potential to improve the health outcomes of people as it increases handwashing and use of soap, which are crucial in breaking the transmission cycle of infections. The findings of our study point to many advantages of tippy-taps over other hand washing station technologies.
These advantages include inexpensive to construct as it uses local materials, easy to construct, entertaining for children, water economical and convenient to use as it is usually constructed near the toilet so that people can easily wash their hands after using the toilet.

In addition, the findings of this study indicate that tippy-taps have a higher likelihood of being adopted by participants. Our study findings show that the majority of participants who constructed their tippy-taps were still using them even after the end of interventions or promotional programs $[7,23,27,32,34]$. This is not surprising given that tippy-taps are cost-effective and are made from locally available resources [6, 46]. Evidence points to the following as facilitators of adoption of public health interventions by users: perceived importance of the intervention, availability of resources, affordability, culturally appropriate, easy to use, availability of technical and financial support [47].

Furthermore, although more than three decades have passed since the first tippy-tap was constructed, the findings of our systematic review demonstrate that there is still limited data regarding its use and effectiveness. Only a few studies have specifically evaluated tippy-tap as an intervention. Out of the 20 studies included in this study, only three were experimental studies [22, 23, 34]. Out of these three experimental studies, only one [34] specifically evaluated the effectiveness of the tippy-tap in preventing stomach pain episodes. In the other two studies [22, 23], tippy-taps were part of a combined water and hygiene interventions that were evaluated together. While Zhang and colleagues [34] reported that tippy-taps were effective in reducing stomach pains episodes among the participants in the treatment group, the study lacked information regarding blinding of participants and measures of fidelity which put to question the validity and reliability of the findings.

The findings of this review suggest that there is a dearth of literature on tippy-tap enabling technology especially on the promotion of handwashing practices. The history of tippy-tap dates to 1980s, but the first peerreviewed article was published in 1994 [25] . Thereafter, a gap ensued until 2011 when Biran and colleagues published the next paper on tippy-tap [7]. Our search strategy indicated that the latest articles in this field were published in 2019 [21, 31] while the remaining studies were conducted between 2011 and 2019.

\section{Limitations of the study}

This review is not without limitations. First, our systematic review only included studies that were conducted in English. This may have introduced bias to the findings of the study as some studies published in other languages may have had information that could be useful in answering the research question. Second, the study was 
limited to poor resource countries limiting the generalisation of the findings to other settings. Notwithstanding these limitations, all the studies except two, scored high $(\geq 75 \%)$ on quality appraisal using MMAT with eight articles scoring $100 \%$. This entails that the majority of the studies included in this review were of moderate or strong quality.

\section{Implications of the study findings for practice, research and policy}

The findings of this systematic review of literature inform practitioners, policy makers and researchers about the use, adoption, benefits, and effectiveness of tippy-taps in resource limited countries. The tippy-tap technology is one of the interventions that people working in the field should promote in resource-limited settings where the majority of people fetch water from community boreholes or wells which are far from their houses. Tippy-taps are cheap, easy to construct, entertaining to children, and easy to adopt which make them suitable hand washing promotion intervention in resource poor countries where the prevalence of waterborne and other infectious diseases is high. Public health care workers, Governments, non-governmental organisations, and other stakeholders are encouraged to take a leading role in promoting the use of tippy-taps to people through public campaigns. The campaigns may target schools, churches, communities, and hospitals where majority of the people can be reached. In addition, use of mass media such as radio and television could also be used to educate people about the importance of tippy-taps. Trainings for community volunteers are also needed to empower them with information on how they can support communities to build their own tippy-taps. Another important issue to consider is that we only identified one study that evaluated the effectiveness of the tippy-taps. Moreover, the study had some methodological problems that impacted on the validity and reliability of the findings. Thus, evidence on the effectiveness of tippy-taps in preventing infectious disease is still limited. Rigorous interventional studies with fidelity measures are needed to evaluate the effectiveness of tippytaps in reducing waterborne and other infectious diseases. Furthermore, although schools are places where children spend much of their time, interact with others and easily get or transmit infections, only five studies [18, 24, 28, 34] included in this review had schools as a study setting. Future studies conducted in school settings are therefore necessary. Government policies that can promote the use of tippy-taps by providing subsidies or empowering communities and households through trainings to build and use tippy-taps are also needed (Hayes et al. 2019).

\section{Conclusion}

Tippy-taps have great potential to improve health outcomes of people living in resource-limited settings where waterborne diseases are common. However, with limited data, it is difficult to ascertain how common tippy-taps are within the community or how effective they are in reducing infections associated with poor hand hygiene. More prevalence and experimental studies are warranted to provide a good understanding of the use, adoption, and effectiveness of tippy-taps. To the best of the authors' knowledge, this is the first systematic review to assess the use, adoption, and effectiveness of tippy-tap handwashing station in promoting hand hygiene practices in a resource-limited setting.

\section{Supplementary information}

Supplementary information accompanies this paper at https://doi.org/10. 1186/s12889-020-09101-w.

Additional file 1: Supplementary material A. Standardised data extraction form

\section{Abbreviations}

AMED: AMED Allied and Complementary Medicine; CINAHL: Cumulative Index of Nursing and Allied Health Literature; COCHRANE: Cochrane database of systematic reviews; COVID-19: Coronavirus disease 19; DOAJ: The Directory of Open Access Journals; EMBASE: Excerpta Medica dataBASE; MMAT: The Mixed Methods Appraisal Tool; PRISMA: Preferred Reporting Items for Systematic Reviews and Meta-Analyses; PROSPERO: The International Prospective Register of Systematic Reviews;

PsycINFO: Psychological Information Database; UNICEF: The United Nations International Children's Emergency Fund; UNC: University of North Caroline; WHO: World Health Organisation

\section{Acknowledgements}

Not applicable

\section{Authors' contributions}

BMC, FWK \& MZ designed the literature review, wrote the protocol that is published in the PROSPERO and searched the literature. Data analysis was done by all authors who also wrote the manuscrips and contributed to the intellectual revisions and content All authors have read and approved the manuscript.

Funding

There was no funding for this systematic review.

Availability of data and materials

The data and materials used in this systematic review are available from the corresponding author on request.

Ethics approval and consent to participate Not applicable.

Consent for publication

Not applicable.

Competing interests

The authors declare no competing interests.

Author details

${ }^{1}$ St John's Institute for Health, P.O. Box 18, Mzuzu, Malawi. ${ }^{2}$ Mzuzu University, Private Bag 201, Luwinga, Mzuzu 2, Malawi. 
Received: 4 October 2019 Accepted: 12 June 2020

Published online: 26 June 2020

\section{References}

1. UNICEF. The Human Right to Water and Sanitation. Geneva: United Nations; 2015.

2. WHO \& UNICEF. Water, sanitation and hygiene in health care facilities: status in low and middle income countries and way forward. Geneva: WHO; 2015.

3. Center for Disease Control and Prevention: Hand Hygiene in Healthcare Settings. In. USA: Center for Disease Control and Prevention; ND.

4. Hutton G, Haller L, WHO Evaluation of the costs and benefits of water and sanitation improvements at the global level. Geneva: WHO; 2004.

5. UNICEF. Fact sheet: child survival fact sheet: water and sanitation, vol. 2020. USA: UNICEF; 2004.

6. Eshuchi R. Promoting handwashing with soap behaviour in Kenyan schools: learning from puppetry trials among primary school children in Kenya. Doctoral Thesis. Australia: Queensland University of Technology; 2013.

7. Adam B. Enabling technologies for handwashing with soap : a case study on the tippy-tap in Uganda. Uganda: Water and Sanitation Program; 2011.

8. UNICEF Zambia. The programme. Zambia: UNICEF; 2012.

9. How to build a tippy tap for hand washing. Available from http://hip.fhi360. org/file/19778/CC\%20How\%20to\%20Build\%20Tippy\%20Tap\%20FINAL.pdf. [cited 2020 June 16]

10. Pickering AJ, Davis J, Blum AG, Scalmanini J, Oyier B, Okoth G, Breiman RF, Ram PK. Access to waterless hand sanitizer improves student hand hygiene behavior in primary schools in Nairobi, Kenya. Am J Trop Med Hyg. 2013; 89(3):411-8.

11. Morgan P. The Mukombe - Zimbabwe's first "tippy tap": A description of its value and use [https://www.aquamor.info/uploads/3/4/2/5/34257237/the_ mukombe.pdf].

12. UNICEF. Water, Sanitation and Hygiene for School children in Emergencies: A Guidebook for Teachers, vol. 75. USA: UNICEF; 2011.

13. WaterAid. Hygiene framework. London: WaterAid; 2012

14. Mbakaya BC, Zgambo M, Kalembo FW: Use, adoption and effectiveness of tippy-tap handwashing station in promoting hand hygiene practices in resource limited setting. PROSPERO database. 2017;CRD42017074331.

15. Moher D, Liberati A, Tetzlaff J, Altman DG. Preferred reporting items for systematic reviews and meta-analyses: the PRISMA statement. Int J Surg. 2010;8(5):336-41.

16. Abass $K$, Achegbulu A. Financial inclusion and safe sanitation in slum communities of Abuja, Nigeria. In: 41st WEDC International Conference. Nakuru: Kenya Loughborough University; 2018.

17. Aiemjoy K, Gebresillasie S, Stoller NE, Shiferaw A, Tadesse Z, Chanyalew M, Aragie S, Callahan K, Keenan JD. Epidemiology of soil-transmitted helminth and intestinal protozoan infections in preschool-aged children in the Amhara region of Ethiopia. Am J Trop Med Hyg. 2017;96(4):866-72.

18. Bresee S, Caruso B, Sales J, Lupele J, Freeman M. A child is also a teacher': exploring the potential for children as change agents in the context of a school-based WASH intervention in rural eastern Zambia. Health Educ Res. 2016:31(4):521-34

19. Cantrell BL. An evaluation of a water, sanitation, and hygiene program in rural communities outside of Port-Au-Prince, Haiti. Thesis. USA: Georgia State University; 2013.

20. Chisanga HJ. Process evaluation on the implementation of Mwanzo bora nutrition program on the reduction of childhood stunting: a case of Kilolo District in Tanzania. Doctoral Thesis. Tanzania: Mzumbe University; 2018.

21. Chidziwisano K, Tilley E, Malolo R, Kumwenda S, Musaya J, Morse T. Risk factors associated with feeding children under 2 years in rural Malawi-a formative study. Int J Environ Res Public Health. 2019;16(12):2146.

22. Christensen G, Dentz HN, Pickering AJ, Bourdier T, Arnold BF, Colford JM Jr, Null C. Pilot cluster randomized controlled trials to evaluate adoption of water, sanitation, and hygiene interventions and their combination in rural western Kenya. Am J Trop Med Hyg. 2015;92(2):437-47.

23. Contzen N, Meili IH, Mosler H-J. Changing handwashing behaviour in southern Ethiopia: a longitudinal study on infrastructural and commitment interventions. Soc Sci Med. 2015;124:103-14.

24. Dajaan DS, Addo HO, Ojo L, Amegah KE, Loveland F, Bechala BD, Benjamin $\mathrm{BB}$. Hand washing knowledge and practices among public primary schools in the Kintampo municipality of Ghana. Int J Community Med Public Health. 2018;5(6):2205-16.
25. Hurtado E. Rapid assessment procedures in formative research for a communication intervention on water-related hygiene behaviours. Food Nutr Bull. 1994;15(1):1-6.

26. Kamuteera E, Trimmer J. Finding sustainable funding for WASH facility monitoring through nutrient recovery in southwest Uganda. In: 41st WEDC International Conference. Nakuru: Kenya Loughborough University; 2018.

27. Mbuya MN, Tavengwa NV, Stoltzfus RJ, Curtis V, Pelto GH, Ntozini R, Kambarami RA, Fundira D, Malaba TR, Maunze D. Design of an intervention to minimize ingestion of fecal microbes by young children in rural Zimbabwe. Clin Infect Dis. 2015;61(suppl_7):S703-9.

28. Musoke D, Ndejjo R, Halage AA, Kasasa S, Ssempebwa JC, Carpenter DO. Drinking water supply, sanitation, and hygiene promotion interventions in two slum communities in Central Uganda. J Environ Public Health. 2018; 2018

29. Mwakitalima A, Massa K, Seleman A, Kassile T. Scaling up rural sanitation in Tanzania: evidence from the National Sanitation Campaign. J Water Sanit Hyg Dev. 2018;8(2):290-306.

30. Pietropaoli J, Moses P, Forrester $\mathrm{H}$. Using trials of improved practices to shift nutrition and hygiene behaviours in Sierra Leone. Field Exchange 54, vol. 33, 2017.

31. Shukla M. Implementing innovative and sustainable methods to tackle grassroot level problems at anganwadi centers in Virpapura Village, Karnataka (India). J Dental Res Rev. 2018:5(4):139.

32. Singh D, Cumming R, Mohajer N, Negin J. Motivation of community health volunteers in rural Uganda: the interconnectedness of knowledge, relationship and action. Public Health. 2016:136:166-71.

33. Singh D, Negin J, Orach CG, Cumming R. Supportive supervision for volunteers to deliver reproductive health education: a cluster randomized trial. Reprod Health. 2016;13(1):126.

34. Zhang C, Mosa A, Hayward A, Matthews SA. Promoting clean hands among children in Uganda: a school-based intervention using 'tippy-taps'. Public Health. 2013;127(6):586.

35. Pluye P, Robert E, Cargo M, Bartlett G, O'cathain A, Griffiths F, Boardman F, Gagnon M-P, Rousseau M. Proposal: a mixed methods appraisal tool for systematic mixed studies reviews. Montréal: McGill University; 2011. p. 1-8.

36. Boerleider AW, Wiegers TA, Manniën J, Francke AL, Devillé WL. Factors affecting the use of prenatal care by non-western women in industrialized western countries: a systematic review. BMC Pregnancy Childbirth. 2013; 13(1):81.

37. Peek ST, Wouters EJ, van Hoof J, Luijkx KG, Boeije HR, Vrijhoef HJ. Factors influencing acceptance of technology for aging in place: a systematic review. Int J Med Inform. 2014;83(4):235-48.

38. Pieper MJ, van Dalen-Kok AH, Francke AL, van der Steen JT, Scherder EJ, Husebø BS, Achterberg WP. Interventions targeting pain or behaviour in dementia: a systematic review. Ageing Res Rev. 2013;12(4): 1042-55.

39. Popay J, Roberts H, Sowden A, Petticrew M, Arai L, Rodgers M, Britten N Roen K, Duffy S: Guidance on the conduct of narrative synthesis in systematic reviews: A product from the ESRC methods programme Version. Lancaster: Lancaster University 2006, 1(2.1):1018.4643.

40. Noyes J, Booth A, Moore G, Flemming K, Tunçalp Ö, Shakibazadeh E. Synthesising quantitative and qualitative evidence to inform guidelines on complex interventions: clarifying the purposes, designs and outlining some methods. BMJ Glob Health. 2019;4(Suppl 1):e000893.

41. Brewster L, Mountain G, Wessels B, Kelly C, Hawley M. Factors affecting front line staff acceptance of telehealth technologies: a mixed-method systematic review. J Adv Nurs. 2014;70(1):21-33.

42. Dai Y, Livesley J. A mixed-method systematic review of the effectiveness and acceptability of preoperative psychological preparation programmes to reduce paediatric preoperative anxiety in elective surgery. J Adv Nurs. 2018;74(9):2022-37.

43. Mbakaya BC, Lee PH, Lee RL. Hand hygiene intervention strategies to reduce diarrhoea and respiratory infections among schoolchildren in developing countries: a systematic review. Int J Environ Res Public Health. 2017;14(4):371.

44. Stephenson R, Simon C, Finneran C. Community factors shaping early age at first sex among adolescents in Burkina Faso, Ghana, Malawi, and Uganda. J Health Popul Nutr. 2014;32(2):161.

45. WHO. Guidelines on Hand Hygiene in Health Care: First Global Patient Safety Challenge. In: Clean Care is Safer Care. Geneva: WHO; 2019.

46. UNICEF. Soap, toilets and taps: A foundation for healthy children. New York: UNICEF; 2009, 3(4). 
47. Jeffries M, Phipps D, Howard RL, Avery A, Rodgers S, Ashcroft D. Understanding the implementation and adoption of an information technology intervention to support medicine optimisation in primary care: qualitative study using strong structuration theory. BMJ Open. 2017;7(5): e014810.

\section{Publisher's Note}

Springer Nature remains neutral with regard to jurisdictional claims in published maps and institutional affiliations.

Ready to submit your research? Choose BMC and benefit from:

- fast, convenient online submission

- thorough peer review by experienced researchers in your field

- rapid publication on acceptance

- support for research data, including large and complex data types

- gold Open Access which fosters wider collaboration and increased citations

- maximum visibility for your research: over $100 \mathrm{M}$ website views per year

At $\mathrm{BMC}$, research is always in progress.

Learn more biomedcentral.com/submissions 